\title{
ON THE GAUSSIAN LIMITING DISTRIBUTION OF LATTICE POINTS IN A PARALLELEPIPED
}

\author{
Mordechay B. LeVin \\ Dedicated to the memory of Professor Pierre Liardet
}

\begin{abstract}
Let $\Gamma \subset \mathbb{R}^{s}$ be a lattice obtained from a module in a totally real algebraic number field. Let $\mathcal{R}(\boldsymbol{\theta}, \mathbf{N})$ be the error term in the lattice point problem for the parallelepiped $\left[-\theta_{1} N_{1}, \theta_{1} N_{1}\right] \times \cdots \times\left[-\theta_{s} N_{s}, \theta_{s} N_{s}\right]$. In this paper, we prove that $\mathcal{R}(\boldsymbol{\theta}, \mathbf{N}) / \sigma(\mathcal{R}, \mathbf{N})$ has a Gaussian limiting distribution as $N \rightarrow \infty$, where $\boldsymbol{\theta}=\left(\theta_{1}, \ldots, \theta_{s}\right)$ is a uniformly distributed random variable in $[0,1]^{s}$, $N=N_{1} \cdots N_{s}$ and $\sigma(\mathcal{R}, \mathbf{N}) \asymp(\log N)^{(s-1) / 2}$. We obtain also a similar result for the low discrepancy sequence corresponding to $\Gamma$. The main tool is the $S$-unit theorem.
\end{abstract}

\section{Communicated by Werner Georg Nowak}

\section{Introduction}

\subsection{Preliminaries}

In 1992, J. Beck (see Be1-Be3] $)^{1}$ discovered a very surprising phenomenon of randomness of the sequence $\{n \sqrt{2}\}_{n \geq 1}$ and the lattice

$$
\begin{aligned}
& \left\{(n, n \sqrt{2}+m) \mid(n, m) \in \mathbb{Z}^{2}\right\}: \\
& \quad \operatorname{vol}\left\{(x, y, z) \in[0,1)^{3}: \frac{\sum_{n=0}^{[x N]}\left(\mathbb{1}_{[0, y)}(\{n \sqrt{2}+z\})-y\right)}{c_{1} \sqrt{\log N}}<t\right\} \rightarrow \Phi(t)
\end{aligned}
$$

as $N \rightarrow \infty$, where $\Phi(t)=\frac{1}{\sqrt{2 \pi}} \int_{-\infty}^{t} e^{-u^{2} / 2} d u, \mathbb{1}_{\mathcal{O}}(x)$ is the indicator function of $\mathcal{O}, c_{1}>0$ and $\{v\}$ is the fractional part of $v$.

2010 Mathematics Subject Classification: Primary 11P21, 11K38, 11R80; Secondary $60 \mathrm{~F} 05$.

Keywords: lattice points problem, low discrepancy sequences, totally real algebraic number field, central limit theorem.

${ }^{1}$ The results of this paper were announced in Le1], Le2]. 
According to $\mathrm{Be} 2$, p. 41], the generalizations of this result to the multidimensional case for a Kronecker's lattice is very difficult because of problems connected to Littlewood's conjecture:

$$
\underline{\lim _{n \rightarrow \infty}} n \ll n \alpha \gg \ll n \beta \gg=0
$$

for all reals $\alpha, \beta$, where $\ll x \gg=\min (\{x\}, 1-\{x\})$.

In this paper, in order to avoid these problems, we consider a lattice $\Gamma$ obtained from a module in a totally real algebraic number field. We prove the Central Limit Theorem (abbreviated CLT) for the number of points in a parallelepiped. We obtain also a similar result for low discrepancy sequences corresponding to $\Gamma$ (see [Le2]). For related questions and generalizations, see [Le3]. In a forthcoming paper, we will generalize results from Be2 to the cases of $s$-dimensional Halton's sequences (for 1-dimensional case see [LeMe]), $(t, s)$-sequences, and admissible lattices (see the definition below).

\subsection{Lattice points}

Let $\mathcal{O} \subset \mathbb{R}^{s}$ be a compact region, vol $\mathcal{O}$ its volume, $t \mathcal{O}$ its dilatation of $\mathcal{O}$ by a factor $t>0$, and let $t \mathcal{O}+\mathbf{x}$ be the translation of $t \mathcal{O}$ by a vector $\mathbf{x} \in \mathbb{R}^{s}$. Let $\Gamma \subset \mathbb{R}^{s}$ be a lattice, i.e., a discrete subgroup of $\mathbb{R}^{s}$ with a compact fundamental set $\mathbb{R}^{s} / \Gamma$, and denote $\operatorname{det} \Gamma=\operatorname{vol}\left(\mathbb{R}^{s} / \Gamma\right)$. Let

$$
\mathcal{N}(\mathcal{O}, \Gamma)=\operatorname{card}(\mathcal{O} \cap \Gamma)=\sum_{\gamma \in \Gamma} \mathbb{1}_{\mathcal{O}}(\gamma)
$$

be the number of points of $\Gamma$ lying inside $\mathcal{O}$. We define the error $\mathcal{R}(\mathcal{O}+\mathbf{x}, \Gamma)$ by

$$
\mathcal{N}(\mathcal{O}+\mathbf{x}, \Gamma)=\operatorname{vol} \mathcal{O} \cdot(\operatorname{det} \Gamma)^{-1}+\mathcal{R}(\mathcal{O}+\mathbf{x}, \Gamma) .
$$

We define the norm of $\mathbf{x}=\left(x_{1}, \ldots, x_{s}\right)$ by $\mathrm{Nm}(\mathbf{x})=x_{1} x_{2} \cdots x_{s}$. The lattice $\Gamma \subset \mathbb{R}^{s}$ is admissible if

$$
\operatorname{Nm} \Gamma=\inf _{\gamma \in \Gamma \backslash\{0\}}|\operatorname{Nm}(\gamma)|>0 .
$$

Let $\mathcal{K}$ be a totally real algebraic number field of degree $s \geq 2$, and let $\sigma$ be the canonical embedding of $\mathcal{K}$ in the Euclidean space $\mathbb{R}^{s}, \sigma: \mathcal{K} \ni \xi \rightarrow$ $\sigma(\xi)=\left(\sigma_{1}(\xi), \ldots, \sigma_{s}(\xi)\right) \in \mathbb{R}^{s}$, where $\left\{\sigma_{j}\right\}_{j=1}^{s}$ are the embeddings of $\mathcal{K}$ in $\mathbb{R}$. Let $N_{\mathcal{K} / \mathbb{Q}}(\xi)$ be the norm of $\xi \in \mathcal{K}$. By [BS, p. 404]

$$
N_{\mathcal{K} / \mathbb{Q}}(\xi)=\sigma_{1}(\xi) \cdots \sigma_{s}(\xi), \quad \text { and } \quad\left|N_{\mathcal{K} / \mathbb{Q}}(\alpha)\right| \geq 1
$$

for all algebraic integers $\alpha \in \mathcal{K} \backslash\{0\}$. Thus $|\operatorname{Nm}(\sigma(\xi))|=\left|N_{\mathcal{K} / \mathbb{Q}}(\xi)\right|$. Let $M$ be a full $\mathbb{Z}$-module in $\mathcal{K}$, and let $\Gamma_{M}$ be the lattice corresponding to $M$ under the embedding $\sigma$. It is known that the set $M^{\perp}$ of all $\beta \in \mathcal{K}$, for which $\operatorname{Tr}_{\mathcal{K} / \mathbb{Q}}(\alpha \beta) \in \mathbb{Z}$ for all $\alpha \in M$, is also a full $\mathbb{Z}$ - module (the dual of the module $M$ ) of the 


\section{GAUSSIAN LIMITING DISTRIBUTION OF LATTICE POINTS IN PARALLEPIDED}

field $\mathcal{K}$ (see [BS, p. 94]). Recall that the dual lattice $\Gamma_{M}^{\perp}$ consists of all vectors $\gamma^{\perp} \in \mathbb{R}^{s}$ such that the inner product $\left\langle\gamma^{\perp}, \gamma\right\rangle$ belongs to $\mathbb{Z}$ for each $\gamma \in \Gamma$. Hence $\Gamma_{M^{\perp}}=\Gamma_{M}^{\perp}$. Let $\left(C_{M}\right)^{-1}>0$ be an integer such that $\left(C_{M}\right)^{-1} \gamma$ are algebraic integers for all $\gamma \in M \cup M^{\perp}$. Hence

$$
\min \left(\mathrm{Nm} \Gamma_{M}, \mathrm{Nm} \Gamma_{M}^{\perp}\right) \geq C_{M}^{s} .
$$

Therefore $\Gamma_{M}$ and $\Gamma_{M \perp}$ are admissible lattices. In the following we will use the notation $\Gamma=\Gamma_{M}$.

We note that the problem considered in this paper is closely connected with quantum chaos theory. By [B] and [Ma, the problem about the number of eigenvalues of an operator in a quantum system in a large interval $[0, t]$ leads to the problem of counting the number of lattice points in a domain $t \mathcal{O}$. For example, the particular case of the famous Berry-Tabor conjecture (see, e.g., Ma ) consists of the assertion that the number of lattice points in the thin domain $(t+1 / t) \mathcal{O} \backslash t \mathcal{O}$ tends to the Poisson distribution (for the case of 'generic' lattice), where $t$ is a uniformly distributed random variable in $[0, L]$ and $L \rightarrow \infty$ (see, e.g., [B], [Ma], and [Si]).

\subsection{Low discrepancy sequences}

Let $\left(\left(\beta_{n, N}\right)_{n=0}^{N-1}\right)$ be an $N$-point set in the $s$-dimensional unit cube $[0,1)^{s}$, $\mathcal{O}=\left[0, y_{1}\right) \times \cdots \times\left[0, y_{s}\right) \subseteq[0,1)^{s}$,

$$
\Delta\left(\mathcal{O},\left(\beta_{n, N}\right)_{n=0}^{N-1}\right)=\sum_{0 \leq n \leq N-1} \mathbb{1}_{\mathcal{O}}\left(\beta_{n, N}\right)-y_{1} y_{2} \cdots y_{s} N .
$$

We define the $L^{\infty}$ and $L^{2}$ discrepancy of an $N$-point set $\left(\beta_{n, N}\right)_{n=0}^{N-1}$ by

$$
\begin{aligned}
D\left(\left(\beta_{n, N}\right)_{n=0}^{N-1}\right) & =\sup _{0<y_{1}, \ldots, y_{s} \leq 1}\left|\frac{1}{N} \Delta\left(\mathcal{O},\left(\beta_{n, N}\right)_{n=0}^{N-1}\right)\right| \\
D_{2}\left(\left(\beta_{n, N}\right)_{n=0}^{N-1}\right) & =\left(\int_{[0,1]^{s}}\left|\frac{1}{N} \Delta\left(\mathcal{O},\left(\beta_{n, N}\right)_{n=0}^{N-1}\right)\right|^{2} \mathrm{dy}_{1} \cdots \mathrm{dy}_{\mathrm{s}}\right)^{1 / 2},
\end{aligned}
$$

respectively. In 1954, Roth proved that there exists a constant $C>0$, such that

$$
N D_{2}\left(\left(\beta_{n, N}\right)_{n=0}^{N-1}\right)>C(\ln N)^{\frac{s-1}{2}}
$$

for all $N$-point sets $\left(\beta_{n, N}\right)_{n=0}^{N-1}$.

Definition. A sequence $\left(\beta_{n}\right)_{n \geq 0}$ is of low discrepancy (abbreviated l.d.s.) if

$$
D\left(\left(\beta_{n}\right)_{n=0}^{N-1}\right)=O\left(N^{-1}(\ln N)^{s}\right) \quad \text { as } \quad N \rightarrow \infty .
$$


A sequence of point sets $\left(\left(\beta_{n, N}\right)_{n=0}^{N-1}\right)_{N=1}^{\infty}$ is of low discrepancy (abbreviated l.d.p.s.) if

$$
D\left(\left(\beta_{n, N}\right)_{n=0}^{N-1}\right)=O\left(N^{-1}(\ln N)^{s-1}\right) \quad \text { as } \quad N \rightarrow \infty .
$$

For examples of l.d.s. and l.d.p.s. see [BC] and [DrTi]. In [Fr], Frolov constructed a low discrepancy point set from a module in a totally real algebraic number field (see also By, $[\mathrm{Skr}]$ ). Using this approach, we proposed in [Le2] the following construction of l.d.s. :

According to (1.4), $\left|\mathrm{Nm}\left(\gamma^{(1)}-\gamma^{(2)}\right)\right| \geq C_{M}^{s}$ for different points $\gamma^{(1)}, \gamma^{(2)} \in \Gamma$. Hence, there are no two different points $\gamma^{(1)}, \gamma^{(2)} \in \Gamma$ with $\gamma_{s}^{(1)}=\gamma_{s}^{(2)}$. It follows that the set $W_{\mathbf{x}}=((\mathbf{x}, 0)+\Gamma) \cap[0,1)^{s-1} \times(-\infty, \infty)$ with $\mathbf{x} \in[0,1)^{s-1}$ can be enumerated by a sequence $\left(z_{\mathbf{x}, k}, z_{s}(\mathbf{x}, k)\right)_{k=-\infty}^{+\infty}$ in the following way:

$$
z_{\mathbf{x}, 0}=\mathbf{x}, z_{s}(\mathbf{x}, 0)=0, \quad z_{\mathbf{x}, k} \in[0,1)^{s-1} \text { and } z_{s}(\mathbf{x}, k)<z_{s}(\mathbf{x}, k+1) \in \mathbb{R}
$$

for $k \in \mathbb{Z}$. We see that there exists a unique $\left(w, y_{s}\right) \in W_{\mathbf{x}}$ with

$$
y_{s}=\min \left\{v>0 \mid \exists w \in[0,1)^{s-1}, \text { such that }(w, v) \in W_{\mathbf{x}}\right\} .
$$

Let $\mathcal{T}(\mathbf{x})=w$. In Le2, we proved that $\mathcal{T}(\mathbf{x})$ is an ergodic transformation with respect to the Lebesgue measure on $[0,1)^{s-1}, \mathcal{T}^{k}(\mathbf{x})=z_{k}(\mathbf{x})$ for $k \in \mathbb{Z}$, and $\left(T^{k}(x)\right)_{n \geq 1}$ is of low discrepancy, i.e., $D_{N}\left(\left(T^{k}(x)\right)_{n \geq 1}\right)=O\left(\ln ^{s} N\right)$. In [Le4, we proved that this estimate cannot be improved.

In Theorem 2, we will prove that there exists a sequence $\left(\beta_{k}\right)_{k \geq 0}$ such that the triangular array of random variables $\left(\mathbb{1}_{\left[0, y_{1}\right) \times \cdots \times\left[0, y_{s-1}\right)}\left(\beta_{k}\right)\right)_{k=0}^{\left[y_{s} \bar{N}\right]-1}$ satisfies the CLT with an extremely small (by order of magnitude) standard deviation (see Roth's lower bound (1.6) ). We will take $\beta_{k}=\mathcal{T}^{k}(\mathbf{x}), k=0,1, \ldots$

\subsection{Statement of the results}

We consider the probability space $\left([0,1]^{s}, \lambda, B\left([0,1]^{s}\right)\right)$ with Lebesgue's measure $\lambda$. Hence, we have the following formula for the expectation:

$$
\mathbf{E}[f(\boldsymbol{\theta})]=\int_{[0,1]^{s}} f(\boldsymbol{\theta}) \mathrm{d} \boldsymbol{\theta} .
$$

We define the variation of $f$ by $\operatorname{Var}(f)=\mathbf{E}\left[f^{2}\right]-(\mathbf{E}[f])^{2}$. Let $\mathbb{K}_{s}=[-1 / 2,1 / 2)^{s}$,

$$
\begin{gathered}
\mathbf{N}=\left(N_{1}, \ldots, N_{s}\right), N_{i}>0,(i=1, \ldots, s), N=N_{1} N_{2} \cdots N_{s}, \\
\left(x_{1}, \ldots, x_{s}\right) \cdot\left(y_{1}, \ldots, y_{s}\right)=\left(x_{1} y_{1}, \ldots, x_{s} y_{s}\right), \\
\left(x_{1}, \ldots, x_{s}\right) \cdot \mathcal{O}=\left\{\left(x_{1}, \ldots, x_{s}\right) \cdot\left(y_{1}, \ldots, y_{s}\right):\left(y_{1}, \ldots, y_{s}\right) \in \mathcal{O}\right\}
\end{gathered}
$$

and $n=\left[\log _{2} N\right]+1$. 


\section{GAUSSIAN LIMITING DISTRIBUTION OF LATTICE POINTS IN PARALLEPIDED}

TheOREM 1. With the notations as above, there exist $w_{2}>w_{1}>0$ such that

$$
\sup _{t, \mathbf{x}}\left|\operatorname{vol}\left\{\boldsymbol{\theta} \in \mathbf{I}^{s}: \frac{\mathcal{R}\left(\boldsymbol{\theta} \cdot \mathbf{N} \cdot \mathbb{K}_{s}+\mathbf{x}, \Gamma\right)}{w(\mathbf{N}, \mathbf{x}) n^{(s-1) / 2}}<t\right\}-\Phi(t)\right|=O\left(n^{-1 / 15}\right)
$$

as $N \rightarrow \infty$, with $w(\mathbf{N}, \mathbf{x})=\operatorname{Var}^{1 / 2}\left(\mathcal{R}\left(\boldsymbol{\theta} \cdot \mathbf{N} \cdot \mathbb{K}_{s}+\mathbf{x}, \Gamma\right)\right) n^{-(s-1) / 2} \in\left[w_{1}, w_{2}\right]$, $\mathbf{I}=[0,1)$ for all $\mathbf{x} \in[0,1)^{s-1}$.

Theorem 2. Let $\mathcal{O}=\left[0, y_{1}\right) \times \cdots \times\left[0, y_{s-1}\right)$. Then there exist $w_{2}>w_{1}>0$ such that

$$
\begin{aligned}
& \sup _{t}\left|\operatorname{vol}\left\{\mathbf{y} \in \mathbf{I}^{s}, \mathbf{x} \in \mathbf{I}^{s-1}: \frac{\Delta\left(\mathcal{O},\left(\mathcal{T}^{k}(\mathbf{x})\right)_{k=0}^{\left[y_{s} N\right]-1}\right)}{v(N, \mathbf{x}) n^{(s-1) / 2}}<t\right\}-\Phi(t)\right|=O\left(n^{-1 / 15}\right) \\
& \text { as } N \rightarrow \infty \text {, with } v(N, \mathbf{x})=\operatorname{Var}^{1 / 2}\left(\Delta\left(\mathcal{O},\left(\mathcal{T}^{k}(\mathbf{x})\right)_{k=0}^{\left[y_{s} N\right]-1}\right)\right) n^{-(s-1) / 2} \in\left[w_{1}, w_{2}\right] \text {. }
\end{aligned}
$$

Throughout the paper, $O$-constants do not depend on $\mathbf{x}, \boldsymbol{\theta}$ and $\mathbf{N}$.

REMARK. Let $K\left(r_{1}, r_{2}\right)$ be an algebraic number field with signature $\left(r_{1}, r_{2}\right), r_{1}+$ $2 r_{2}=s, \Gamma=\Gamma\left(M, r_{1}, r_{2}\right) \subset \mathbb{R}^{s}$ a lattice obtained from a module $M$ in $K\left(r_{1}, r_{2}\right)$, $\mathbf{N}=\left(N_{1}^{\prime}, \ldots, N_{r_{1}}^{\prime}, N_{1}, \ldots, N_{r_{2}}\right) \in \mathbb{Z}_{+}^{r_{1}+r_{2}}, \gamma=\left(\gamma_{1}^{\prime}, \ldots, \gamma_{r_{1}}^{\prime}, \gamma_{1}, \ldots, \ldots, \gamma_{r_{2}}\right) \in \mathbb{R}^{s}$ $\left(\gamma_{i}^{\prime} \in \mathbb{R}, \gamma_{j} \in \mathbb{R}^{2}, i=1, \ldots, r_{1}, j=1, \ldots, r_{2}\right), \mathbf{y}=\left(y_{1}^{\prime}, \ldots \ldots, y_{r_{1}}^{\prime}, y_{1}, \ldots, y_{r_{2}}\right)$, $V=\mathbb{R}^{s} / \Gamma,(\mathbf{y}, \mathbf{x})$ a uniformly distributed random variable in $[0,1]^{r_{1}+r_{2}} \times V$,

$$
G(\mathbf{N})=\prod_{i=1}^{r_{1}}\left[-N_{i} y_{i}, N_{i} y_{i}\right] \prod_{j=1}^{r_{2}}\left\{z \in \mathbb{R}^{2} \quad|z| \leq N_{j} y_{j}\right\}
$$

and let

$$
\begin{aligned}
& \xi_{1}(\mathbf{N})=\sum_{\gamma \in \Gamma+\mathbf{x}} \mathbf{1}_{G(\mathbf{N})}(\boldsymbol{\gamma}), \\
& \xi_{2}(\mathbf{N})=\sum_{\gamma \in \Gamma+\mathbf{x}} \mathbf{1}_{G(\mathbf{N})}(\gamma) \prod_{j=1}^{r_{2}} \sqrt{N_{j}^{2} y_{j}^{2}-\gamma_{j}^{2}} .
\end{aligned}
$$

In a forthcoming paper, we will prove CLT for the multisequence $\xi_{i}(\mathbf{N})$, where $i=1$ if $r_{2} \geq 2$ and $i=2$ if $r_{2}=1, r_{1} \geq 1$. The case $r_{2}=1, r_{1}=0$ was investigated earlier by Hughes and Rudnick [HuRu].

Let us describe the main steps of the proof of Theorem 1:

In Subsection 2.1, we use the Poisson summation formula and the standard trick of 'smoothing'. This allows us to express the error $\dot{\mathcal{R}}$ in terms on absolutely convergent Fourier series. Let $\eta_{1}, \ldots, \eta_{s-1}$ be a set of fundamental units of the field $\mathcal{K}$, and let $A_{1}, \ldots, A_{s-1}$ be a set of the appropriate toral automorphisms. Let $F_{1} \subset \mathbb{R}^{s}$ be a fundamental domain for the field $\mathcal{K}$. 
According to [BS, p. 112] for all $\gamma \in \Gamma^{\perp}$ there exist $\gamma_{1} \in F_{1} \cap \Gamma^{\perp}$ such that $\gamma=\gamma_{1} \eta_{1}^{k_{1}} \ldots \eta_{s-1}^{k_{s-1}}$ for some $k_{1}, \ldots k_{s-1} \in \mathbb{Z}$. By (2.1.2) and (2.1.6), we get that the main part of the error $\dot{\mathcal{R}}$ can be expressed as a sum of the form $\sum_{k_{1}=-n}^{n} \cdots \sum_{k_{s-1}=-n}^{n} f_{\mathbf{N}, \tau, \mathbf{x}}\left(A_{1}^{k_{1}} \cdots A_{s-1}^{k_{s-1}} \boldsymbol{\theta}\right)$. The function $f_{\mathbf{N}, \tau, \mathbf{x}}$ does not comply with conditions of [Le3, Theorem 5]. Hence, we cannot apply [Le3, Theorem 5] to immediately obtain Theorem [1. Therefore, we should reprove [Le3, Theorem 5]. In [Le3, Theorem 5], we use the moment method. In this paper, we use the martingale method. But the main idea of this article is the same as in [Le3, Theorem 5] and is as follows:

In order to prove the central limit theorem, it is sufficient to calculate the upper bound of the number of solutions of the exponential Diophantine equation

$$
\sum_{i=1}^{d} \beta_{i} \eta_{1}^{k_{i, 1}} \cdots \eta_{s-1}^{k_{i, s-1}}=0, \quad k_{i, j} \in\{-n, \ldots, 0, \ldots, n\}, \quad \beta_{i} \in \mathcal{K},
$$

$i=1, \ldots, d, j=1, \ldots, s-1$, where $d \rightarrow \infty$ in [Le3, Theorem 5], and $d=4$ in this paper. We apply the $S$-unit theorem to obtain this bound.

In Subsection 2.2, we consider a dyadic decomposition of the domain of summation $\Gamma^{\perp}$ of the Fourier series of the error $\dot{\mathcal{R}}$ in the regions $\Gamma^{\perp} \cap B_{\mathbf{k}, j}$, where $B_{\mathbf{k}, j}=\left[2^{k_{1}}, 2^{k_{1}}+1\right) \times \cdots \times\left[2^{k_{s-1}}, 2^{k_{s-1}}+1\right) \times\left[j 2^{k_{s}}, j 2^{k_{s}}+1\right) \mathbf{k} \in \mathbb{Z}^{s}$, $k_{1}+\cdots+k_{s}=0, j \in \mathbb{Z}$. In [Le3], we used a more natural decomposition $\Gamma^{\perp}=$ $\cup_{k_{1}, \ldots, k_{s-1} \in \mathbb{Z}}\left(F_{1} \cup \Gamma^{\perp}\right) \eta_{1}^{k_{1}} \cdots \eta_{s-1}^{k_{s-1}}$. However, the dyadic decomposition is more appropriate to construct the martingale difference area $\left(\left(\mathcal{A}\left(\dot{G}_{k}\right)\right)_{k=1}^{n}\right.$, see (2.3.8), (2.4.1) and (2.8.1)). In addition, this allows us to compute the volume of the most important domains of the lattice $\Gamma^{\perp}$, such as $B_{\mathbf{k}, j} \cap\left\{\boldsymbol{\gamma} \in \Gamma^{\perp} \mid \mathrm{Nm}(\boldsymbol{\gamma}) \leq n^{1 / 2}\right\}$.

In Subsection 2.3, we cite two famous Diophantine results - the $S$-unit theorem [ESS] (see Theorem A) and lower bounds for linear forms in logarithms of algebraic numbers see [BW, Theorem B] We decompose the domain of summation $\Gamma^{\perp}$ to five parts: $\Gamma^{\perp}=G_{1} \cup \cdots \cup G_{5}$. Next we apply Theorem B to the main part $G_{1}$.

In Subsection 2.4, we find upper bounds on the variance of the error $\dot{\mathcal{R}}$ ( $\dot{\mathcal{R}}=$ $\left.\mathcal{A}\left(G_{1}\right)+\cdots+\mathcal{A}\left(G_{5}\right)\right)$. It is clear that it is sufficient to compute separately the variance of $\mathcal{A}\left(G_{i}\right), i=1, \ldots, 5$ (see Lemma 7 - Lemma 10).

In Subsection 2.5, we obtain (in Lemma 13) lower bounds on the variance of $\dot{\mathcal{R}}$. Lemma 13 is a simple consequence of Lemma 12, The proof of Lemma 12 is based on the admissibility property of the lattice $\Gamma^{\perp}$ and on Minkowski's convex body theorem. 


\section{GAUSSIAN LIMITING DISTRIBUTION OF LATTICE POINTS IN PARALLEPIDED}

In Subsections 2.6 and 2.7, we use the $S$-unit theorem to compute

$$
\sum_{i=1}^{n} E\left[\mathcal{A}^{4}\left(\dot{G}_{i}\right)\right] \text { and } \sum_{i=1}^{n} E\left[\mathcal{A}^{2}\left(\dot{G}_{i}\right)-E\left(\mathcal{A}^{2}\left(\dot{G}_{i}\right)\right)\right]^{2} .
$$

In Subsection 2.8, we prove the martingale property of the sequence

$$
\left(\mathcal{A}\left(\dot{G}_{i}\right)\right)_{i \geq 1} \text {. }
$$

Using Theorem C, we prove CLT for the main part $\mathbb{S}_{n}$ of $\dot{\mathcal{R}}$.

In Subsection 2.9 , we prove that the difference $\dot{\mathcal{R}}-\mathbb{S}_{n}$ is very small, and Theorem 1 follows.

\section{Proofs of theorems}

We will prove Theorem 1 by using the martingale CLT [Mo] (see Theorem C). First we will find the upper and lower bounds of the variance of the error $\dot{\mathcal{R}}$. Next we approximate $\dot{\mathcal{R}}$ by a martingale difference area $\dot{\mathcal{R}}=\mathcal{A}\left(\dot{G}_{1}\right)+\cdots+\mathcal{A}\left(\dot{G}_{n}\right)$.

According to Theorem $\mathrm{C}$, in order to prove the CLT, it is sufficient to show that $\mathbb{W}_{n}+\mathbb{A}_{n}=o(1)$ for $n \rightarrow \infty$. By Lemma 19, it is sufficient to calculate the upper bound of $E\left[\mathcal{A}^{4}\left(\dot{G}_{i}\right)\right]$ (resp. $E\left[\mathcal{A}^{2}\left(\dot{G}_{i}\right)-E\left(\mathcal{A}^{2}\left(\dot{G}_{i}\right)\right)\right]^{2}$ ) to get $\mathbb{W}_{n}$, see (2.8.13) (resp. $\mathbb{A}_{n}$, see (2.8.16) and (2.8.20) $)$. We calculate $E\left[\mathcal{A}^{4}\left(\dot{G}_{i}\right)\right]$ in Lemma 14 and $E\left[\mathcal{A}^{2}\left(\dot{G}_{i}\right)-E\left(\mathcal{A}^{2}\left(\dot{G}_{i}\right)\right)\right]^{2}$ in Lemma 16.

The main part of evidence in both of these lemmas is to estimate the number of solutions of an exponential Diophantine equation similar to (1.9) (see (2.6.7), (2.7.1) and (2.7.7) ). We get these estimates by using the $S$-unit theorem.

\subsection{Poisson summation formula}

We shall need the Poisson summation formula:

$$
\operatorname{det} \Gamma \sum_{\boldsymbol{\gamma} \in \Gamma} f(\boldsymbol{\gamma}-X)=\sum_{\boldsymbol{\gamma} \in \Gamma^{\perp}} \widehat{f}(\boldsymbol{\gamma}) e(\langle\boldsymbol{\gamma}, \mathbf{x}\rangle),
$$

where

$$
\widehat{f}(Y)=\int_{\mathbb{R}^{s}} f(X) e(\langle\mathbf{y}, \mathbf{x}\rangle) \mathrm{d} \mathbf{x}
$$

is the Fourier transform of $f(X)$, and

$$
e(x)=\exp (2 \pi \sqrt{-1} x),\langle\mathbf{y}, \mathbf{x}\rangle=y_{1} x_{1}+\cdots+y_{s} x_{s} .
$$

Formula 2.1.1 holds for functions $f(\mathbf{x})$ with period lattice $\Gamma$ if one of the functions $f$ or $\widehat{f}$ is integrable and belongs to class $C^{\infty}$ (see, e.g., [SW, p. 251]). 
Let $\mathbf{d}=\left(d_{1}, \ldots, d_{s}\right), d_{i} \geq 0(i=1, \ldots, s), \mathcal{O}_{\mathbf{d}}=\left[-d_{1} / 2, d_{1} / 2\right] \times \cdots$ $\cdots \times\left[-d_{s} / 2, d_{s} / 2\right]$, and let $\widehat{\mathbb{1}}_{\mathcal{O}_{\mathbf{d}}}(\gamma)$ be the Fourier transform of the indicator function $\mathbb{1}_{\mathcal{O}_{\mathbf{d}}}(\gamma)$. It is easy to prove that $\widehat{\mathbb{1}}_{\mathcal{O}_{\mathbf{d}}}(\mathbf{0})=d_{1} d_{2} \cdots d_{s}$ and

$$
\widehat{\mathbb{1}}_{\mathcal{O}_{\mathbf{d}}}(\gamma)=\prod_{i=1}^{s} \frac{e\left(d_{i} \gamma_{i} / 2\right)-e\left(-d_{i} \gamma_{i} / 2\right)}{2 \pi \sqrt{-1} \gamma_{i}}=\prod_{i=1}^{s} \frac{\sin \left(\pi d_{i} \gamma_{i}\right)}{\pi \gamma_{i}}
$$

for $\operatorname{Nm}(\gamma) \neq 0$. We fix a nonnegative function $\omega(\mathbf{x}), \mathbf{x} \in \mathbb{R}^{s}$, of the class $C^{\infty}$, with a support inside the unit ball $|\mathbf{x}| \leq 1$, such that

$$
\int_{\mathbb{R}^{s}} \omega(\mathbf{x}) \mathrm{d} \mathbf{x}=1
$$

We set $\omega_{\tau}(\mathbf{x})=\tau^{-s} \omega\left(\tau^{-1} \mathbf{x}\right), \tau>0$, and

$$
\hat{\omega}(\mathbf{y})=\int_{\mathbb{R}^{s}} e(\langle\mathbf{y}, \mathbf{x}\rangle) \omega(\mathbf{x}) \mathrm{d} \mathbf{x} .
$$

Notice that the Fourier transform $\hat{\omega}_{\tau}(\mathbf{y})=\hat{\omega}(\tau \mathbf{y})$ of the function $\omega_{\tau}(\mathbf{y})$ satisfies the bound

$$
\left|\hat{\omega}_{\tau}(\mathbf{y})\right|<c_{2}(1+\tau|\mathbf{y}|)^{-2 s} .
$$

Lemma 1. There exists a constant $c>0$, such that we have for $N>c$

$$
\left|\mathcal{R}\left(\mathcal{O}_{\boldsymbol{\theta} \cdot \mathbf{N}}+\mathbf{x}, \Gamma\right)-\ddot{\mathcal{R}}\left(\mathcal{O}_{\boldsymbol{\theta} \cdot \mathbf{N}}+\mathbf{x}, \Gamma\right)\right| \leq 2^{s},
$$

where

$$
\ddot{\mathcal{R}}\left(\mathcal{O}_{\boldsymbol{\theta} \cdot \mathbf{N}}+\mathbf{x}, \Gamma\right)=\frac{1}{\operatorname{det} \Gamma} \sum_{\gamma \in \Gamma^{\perp} \backslash \mathbf{0}} \widehat{\mathbb{1}}_{\mathcal{O}_{\boldsymbol{\theta} \cdot \mathbf{N}}}(\boldsymbol{\gamma}) \widehat{\omega}(\tau \boldsymbol{\gamma}) e(\langle\boldsymbol{\gamma}, \mathbf{x}\rangle), \quad \tau=N^{-2} .
$$

Pr o o f. Let $\mathcal{O}_{\boldsymbol{\theta} \cdot \mathbf{N}}^{ \pm \tau}=\left[0, \max \left(0, \theta_{1} N_{1} \pm \tau\right)\right) \times \cdots \times\left[0, \max \left(0, \theta_{s} N_{s} \pm \tau\right)\right)$, and let $\mathbb{1}_{\mathcal{O}}(x)$ be the indicator function of $\mathcal{O}$. We consider the convolutions of the functions $\mathbb{1}_{\mathcal{O}_{\mathbf{N}}^{ \pm \tau}}(\gamma)$ and $\omega_{\tau}(\mathbf{y})$ :

$$
\omega_{\tau} * \mathbb{1}_{\mathcal{O}_{\mathbf{N}}^{ \pm \tau}}(\mathbf{x})=\int_{\mathbb{R}^{s}} \omega_{\tau}(\mathbf{x}-\mathbf{y}) \mathbb{1}_{\mathcal{O}_{\mathbf{N}}^{ \pm \tau}}(\mathbf{y}) \mathrm{d} \mathbf{y} .
$$

It is obvious that the nonnegative functions (2.1.7) are of class $C^{\infty}$ and are compactly supported in $\tau$-neighborhoods of the bodies $\mathcal{O}_{\mathbf{N}}^{ \pm \tau}$, respectively. We obtain

$$
\mathbb{1}_{\mathcal{O}_{\boldsymbol{\theta} \cdot \mathbf{N}}^{-\tau}}(\mathbf{x}) \leq \mathbb{1}_{\mathcal{O}_{\boldsymbol{\theta} \cdot \mathbf{N}}}(\mathbf{x}) \leq \mathbb{1}_{\mathcal{O}_{\boldsymbol{\theta} \cdot \mathbf{N}}^{+\tau}}(\mathbf{x}), \quad \mathbb{1}_{\mathcal{O}_{\boldsymbol{\theta} \cdot \mathbf{N}}^{-\tau}}(\mathbf{x}) \leq \omega_{\tau} * \mathbb{1}_{\mathcal{O}_{\boldsymbol{\theta} \cdot \mathbf{N}}}(\mathbf{x}) \leq \mathbb{1}_{\mathcal{O}_{\boldsymbol{\theta} \cdot \mathbf{N}}^{+\tau}}(\mathbf{x})
$$

Replacing $\mathbf{x}$ by $\gamma-\mathbf{x}$ in (2.1.8) and summing these inequalities over $\gamma \in \Gamma=\Gamma_{M}$, we find from (1.1), that

$$
\mathcal{N}\left(\mathcal{O}_{\boldsymbol{\theta} \cdot \mathbf{N}}^{-\tau}+\mathbf{x}, \Gamma\right) \leq \mathcal{N}\left(\mathcal{O}_{\boldsymbol{\theta} \cdot \mathbf{N}}+\mathbf{x}, \Gamma\right) \leq \mathcal{N}\left(\mathcal{O}_{\boldsymbol{\theta} \cdot \mathbf{N}}^{+\tau}+\mathbf{x}, \Gamma\right),
$$

and 


$$
\mathcal{N}\left(\mathcal{O}_{\boldsymbol{\theta} \cdot \mathbf{N}}^{-\tau}+\mathbf{x}, \Gamma\right) \leq \dot{\mathcal{N}}\left(\mathcal{O}_{\boldsymbol{\theta} \cdot \mathbf{N}}+\mathbf{x}, \Gamma\right) \leq \mathcal{N}\left(\mathcal{O}_{\boldsymbol{\theta} \cdot \mathbf{N}}^{+\tau}+\mathbf{x}, \Gamma\right)
$$

where

$$
\dot{\mathcal{N}}\left(\mathcal{O}_{\boldsymbol{\theta} \cdot \mathbf{N}}+\mathbf{x}, \Gamma\right)=\sum_{\gamma \in \Gamma} \omega_{\tau} * \mathbb{1}_{\mathcal{O}_{\boldsymbol{\theta} \cdot \mathbf{N}}}(\gamma-\mathbf{x})
$$

Hence

$$
\begin{aligned}
-\mathcal{N}\left(\mathcal{O}_{\boldsymbol{\theta} \cdot \mathbf{N}}^{+\tau}+\mathbf{x}, \Gamma\right)+\mathcal{N}\left(\mathcal{O}_{\boldsymbol{\theta} \cdot \mathbf{N}}^{-\tau}+\mathbf{x}, \Gamma\right) & \leq \dot{\mathcal{N}}\left(\mathcal{O}_{\boldsymbol{\theta} \cdot \mathbf{N}}+\mathbf{x}, \Gamma\right)-\mathcal{N}\left(\mathcal{O}_{\boldsymbol{\theta} \cdot \mathbf{N}}+\mathbf{x}, \Gamma\right) \\
& \leq \mathcal{N}\left(\mathcal{O}_{\boldsymbol{\theta} \cdot \mathbf{N}}^{+\tau}+\mathbf{x}, \Gamma\right)-\mathcal{N}\left(\mathcal{O}_{\boldsymbol{\theta} \cdot \mathbf{N}}^{-\tau}+\mathbf{x}, \Gamma\right)
\end{aligned}
$$

Thus

$$
\left|\mathcal{N}\left(\mathcal{O}_{\boldsymbol{\theta} \cdot \mathbf{N}}+\mathbf{x}, \Gamma\right)-\dot{\mathcal{N}}\left(\mathcal{O}_{\boldsymbol{\theta} \cdot \mathbf{N}}+\mathbf{x}, \Gamma\right)\right| \leq \mathcal{N}\left(\mathcal{O}_{\boldsymbol{\theta} \cdot \mathbf{N}}^{+\tau}+\mathbf{x}, \Gamma\right)-\mathcal{N}\left(\mathcal{O}_{\boldsymbol{\theta} \cdot \mathbf{N}}^{-\tau}+\mathbf{x}, \Gamma\right)
$$

Consider the right side of this inequality. We have that $\mathcal{O}_{\boldsymbol{\theta} \cdot \mathbf{N}}^{+\tau} \backslash \mathcal{O}_{\boldsymbol{\theta} \cdot \mathbf{N}}^{-\tau}$ is the union of boxes $\mathcal{O}^{(i)}, i=1, \ldots, 2^{s}-1$, where

$$
\begin{aligned}
\operatorname{vol}\left(\mathcal{O}^{(i)}\right) & \leq \operatorname{vol}\left(\mathcal{O}_{\mathbf{N}}^{+\tau}\right)-\operatorname{vol}\left(\mathcal{O}_{\mathbf{N}}^{-\tau}\right) \leq \prod_{i=1}^{s}\left(N_{i}+\tau\right)-\prod_{i=1}^{s}\left(N_{i}-\tau\right) \\
& \leq N\left(\prod_{i=1}^{s}(1+\tau)-\prod_{i=1}^{s}(1-\tau)\right)<\ddot{c}_{s} N \tau=\ddot{c}_{s} / N, \quad \tau=N^{-2},
\end{aligned}
$$

with some $\ddot{c}_{s}>0$. From (1.4), we get $|\operatorname{Nm}(\boldsymbol{\gamma})| \geq C_{M}^{s}$ for $\gamma \in \Gamma_{M} \backslash \mathbf{0}$. We see $\left|\operatorname{Nm}\left(\gamma_{1}-\gamma_{2}\right)\right| \leq \operatorname{vol}\left(\mathcal{O}^{(i)}+\mathbf{x}\right)<C_{M}^{s} \quad$ for $\quad \gamma_{1}, \gamma_{2} \in \mathcal{O}^{(i)}+\mathbf{x} \quad$ and $\quad N>\ddot{c}_{s} / C_{M}^{s}$. Therefore, the box $\mathcal{O}^{(i)}+\mathbf{x}$ contains at most one point of $\Gamma_{M}$ for $N>\ddot{c} / C_{M}^{s}$. By (2.1.10), we obtain

$$
\left|\dot{\mathcal{N}}\left(\mathcal{O}_{\boldsymbol{\theta} \cdot \mathbf{N}}+\mathbf{x}, \Gamma\right)-\mathcal{N}\left(\mathcal{O}_{\boldsymbol{\theta} \cdot \mathbf{N}}+\mathbf{x}, \Gamma\right)\right| \leq 2^{s}-1, \quad \text { for } \quad N>\ddot{c} / C_{M}^{s}
$$

Let

$$
\dot{\mathcal{R}}\left(\mathcal{O}_{\boldsymbol{\theta} \cdot \mathbf{N}}+\mathbf{x}, \Gamma\right)=\dot{\mathcal{N}}\left(\mathcal{O}_{\boldsymbol{\theta} \cdot \mathbf{N}}+\mathbf{x}, \Gamma\right)-\frac{\operatorname{vol}\left(\mathcal{O}_{\boldsymbol{\theta} \cdot \mathbf{N}}\right)}{\operatorname{det} \Gamma} .
$$

By (2.1.9), we have that $\dot{\mathcal{N}}\left(\mathcal{O}_{\boldsymbol{\theta} \cdot \mathbf{N}}+\mathbf{x}, \Gamma\right)$ is a periodic function of $\mathbf{x} \in \mathbb{R}^{n}$ with the period lattice $\Gamma$. Applying the Poisson summation formula to the series (2.1.9), and bearing in mind that $\widehat{\omega}_{\tau}(\mathbf{y})=\widehat{\omega}(\tau \mathbf{y})$, we obtain from (2.1.6)

$$
\dot{\mathcal{R}}\left(\mathcal{O}_{\boldsymbol{\theta} \cdot \mathbf{N}}+\mathbf{x}, \Gamma\right)=\ddot{\mathcal{R}}\left(\mathcal{O}_{\boldsymbol{\theta} \cdot \mathbf{N}}+\mathbf{x}, \Gamma\right) .
$$

Note that (2.1.5) ensures the absolute convergence of the series (2.1.6) over $\gamma \in \Gamma^{\perp} \backslash\{0\}$. Using (1.2), (2.1.11) and (2.1.12), we get the assertion of Lemma 1. 


\subsection{Dyadic decomposition}

Let $\mathfrak{D}_{M}$ be the ring of coefficients of the full module $M, \mathfrak{U}_{M}$ be the group of units of $\mathfrak{D}_{M}, M_{1}=M, M_{2}=M^{\perp}$, and let $\eta_{k, 1}, \ldots, \eta_{k, s-1}$ be the set of fundamental units of $\mathfrak{U}_{M_{k}}(k=1,2)$. According to the Dirichlet's theorem (see, e.g., [BS, p. 112]), every unit $\eta \in \mathfrak{U}_{M_{k}}$ has a unique representation in the form

$$
\eta=(-1)^{a} \eta_{k, 1}^{a_{1}} \cdots \eta_{k, s-1}^{a_{s-1}}, \quad k=1,2,
$$

where $a_{1}, \ldots a_{s-1}$ are rational integers and $a \in\{0,1\}$. We will denote $\sigma\left(\mathfrak{U}_{M_{k}}\right)$ by the same symbol $\mathfrak{U}_{M_{k}}$.

LEMma 2. Let $y_{1}, \ldots, y_{s}>0$ be reals, $\mathbf{y}=\left(y_{1}, \ldots, y_{s}\right), y=\operatorname{Nm}(\mathbf{y})=y_{1} y_{2} \cdots y_{s}$. Then there exists $\eta_{k}(\boldsymbol{y}) \in \mathfrak{U}_{M_{k}}$ with

$$
y_{i} y^{-1 / s}\left|\sigma_{i}\left(\eta_{k}(\boldsymbol{y})\right)\right| \in\left[1 / c_{0}, c_{0}\right],
$$

where $i=1, \ldots, s, k=1,2$, and

$$
c_{0}=\exp \left(\sum_{k=1,2} \sum_{1 \leq i, j<s}|\ln | \sigma_{i}\left(\eta_{k, j}\right)||\right)>1 \text {. }
$$

Proof. We fix $k \in\{1,2\}$. The matrix $\left(\ln \left|\sigma_{i}\left(\eta_{k, j}\right)\right|\right)_{1 \leq i, j<s}$ is non singular BS, pp. 104, 115]. Hence, there exist reals $b_{1}, \ldots, b_{s-1}$ with

$$
\sum_{1 \leq j<s} b_{j} \ln \left|\sigma_{i}\left(\eta_{k, j}\right)\right|=1 / s \ln y-\ln y_{i}, \quad i=1, \ldots, s-1 .
$$

Taking $a_{j}=\left[b_{j}\right], j=1, \ldots, s-1$ and $\eta_{k}(\boldsymbol{y})=\eta_{k, 1}^{a_{1}} \cdots \eta_{k, s-1}^{a_{s-1}}$, we obtain for $i \in[1, s-1]$,

$$
-\sum_{1 \leq j<s}|\ln | \sigma_{i}\left(\eta_{k, j}\right)|| \leq \ln \left(y_{i} y^{-1 / s}\left|\sigma_{i}\left(\eta_{k}(\boldsymbol{y})\right)\right|\right) \leq \sum_{1 \leq j<s}|\ln | \sigma_{i}\left(\eta_{k, j}\right)|| .
$$

Hence

$$
-\ln c_{0} \leq \ln \left(y_{i} y^{-1 / s}\left|\sigma_{i}\left(\eta_{k}(\mathbf{y})\right)\right|\right) \leq \ln c_{0}, \quad i=1, \ldots, s-1, \quad k=1,2 .
$$

Bearing in mind that $\left|\operatorname{Nm}\left(\eta_{k}(\mathbf{y})\right)\right|=1$ and $y=y_{1} y_{2} \cdots y_{s}$, we get from (2.2.4) and 2.2.3)

$$
\ln \left(y_{s} y^{-1 / s}\left|\sigma_{s}\left(\eta_{k}(\boldsymbol{y})\right)\right|\right)=-\sum_{1 \leq i<s} \ln \left(y_{i} y^{-1 / s}\left|\sigma_{i}\left(\eta_{k}(\boldsymbol{y})\right)\right|\right) \in\left[-\ln c_{0}, \ln c_{0}\right] .
$$

Therefore, the assertion (2.2.2) is true for $i \in[1, s], k=1,2$, and Lemma 2 is proved. 


\section{GAUSSIAN LIMITING DISTRIBUTION OF LATTICE POINTS IN PARALLEPIDED}

We apply this lemma to the vector $\mathbf{y}=\mathbf{N}=\left(N_{1}, \ldots, N_{s}\right)$. Let $N_{i}^{\prime}=$ $N_{i}\left|\sigma_{i}\left(\eta_{1}(\mathbf{N})\right)\right|, i=1, \ldots, s$, and let $\sigma\left(\eta_{1}(\mathbf{N})\right)=\left(\sigma_{1}\left(\eta_{1}(\mathbf{N})\right), \ldots, \sigma_{s}\left(\eta_{1}(\mathbf{N})\right)\right)$. We see that

$\boldsymbol{\gamma} \in \Gamma_{M} \cap\left(\boldsymbol{\theta} \cdot \mathbf{N} \cdot \mathbb{K}^{s}+\mathbf{x}\right) \Leftrightarrow \boldsymbol{\gamma} \cdot \sigma\left(\eta_{1}(\mathbf{N})\right) \in \Gamma_{M} \cap\left(\boldsymbol{\theta} \cdot \mathbf{N}^{\prime} \cdot \mathbb{K}^{s}+\mathbf{x} \cdot \sigma\left(\eta_{1}(\mathbf{N})\right)\right)$.

Hence

$$
\mathcal{N}\left(\boldsymbol{\theta} \cdot \mathbf{N} \cdot \mathbb{K}^{s}+\mathbf{x}, \Gamma_{M}\right)=\mathcal{N}\left(\boldsymbol{\theta} \cdot \mathbf{N}^{\prime} \cdot \mathbb{K}^{s}+\mathbf{x} \cdot \sigma\left(\eta_{1}(\mathbf{N})\right), \Gamma_{M}\right) .
$$

By (1.2), we have

$$
\mathcal{R}\left(\boldsymbol{\theta} \cdot \mathbf{N} \cdot \mathbb{K}^{s}+\mathbf{x}, \Gamma_{M}\right)=\mathcal{R}\left(\boldsymbol{\theta} \cdot \mathbf{N}^{\prime} \cdot \mathbb{K}^{s}+\mathbf{x} \cdot \sigma\left(\eta_{1}(\mathbf{N})\right), \Gamma_{M}\right) .
$$

Therefore, without loss of generality, we can assume that

$$
N_{i} N^{-1 / s} \in\left[1 / c_{0}, c_{0}\right], \quad i=1, \ldots, s .
$$

Now, let $n=\left[\log _{2} N\right]+1$,

$$
\mathbb{F}_{n}^{\prime}=\left\{\gamma \in \Gamma^{\perp} \backslash\{0\}:\left|\gamma_{i}\right||\operatorname{Nm}(\gamma)|^{-1 / s} \in\left[1 / c_{0}, c_{0}\right], i=1, \ldots, s,|\operatorname{Nm}(\gamma)| \leq n^{1 / 2}\right\}
$$

and

$$
\mathbb{F}_{n}=\bigcup_{\gamma \in \mathbb{F}_{n}^{\prime}}\left\{\gamma^{\prime} \in \mathbb{F}_{n}^{\prime}: \gamma_{1}^{\prime}=\max _{\eta \in \mathfrak{U}_{M \perp}, \gamma \cdot \sigma(\eta) \in \mathbb{F}_{n}^{\prime}}(\gamma \cdot \sigma(\eta))_{1}\right\} .
$$

By (1.4), we get that

$$
\text { if } \quad \gamma^{(1)}, \gamma^{(2)} \in \mathbb{F}_{n}, \gamma^{(1)} \neq \gamma^{(2)} \text {, then } \quad \gamma^{(1)} \neq \gamma^{(2)} \cdot \sigma(\eta) \quad \forall \eta \in \mathfrak{U}_{M^{\perp}} .
$$

Lemma 3. Let $a, b \geq 1$ be integers,

$$
\begin{gathered}
\mathbb{G}(a, b)=\left\{\gamma \in \Gamma^{\perp} \backslash\{0\}: \max _{1 \leq i \leq s}\left|\gamma_{i}\right| \in\left(2^{a}, 2^{a+b}\right], \quad|\operatorname{Nm}(\gamma)| \leq n^{1 / 2}\right\}, \\
\mathbb{G}^{\prime}(a, b):=\bigcup_{\gamma \in \mathbb{F}_{n}} \bigcup_{\eta \in \mathfrak{U}(\gamma, a, b)} \gamma \cdot \sigma(\eta),
\end{gathered}
$$

with

Then

$$
\dot{\mathfrak{U}}\left(\gamma^{(0)}, a, b\right)=\left\{\eta \in \mathfrak{U}_{M^{\perp}}: \gamma^{(0)} \cdot \sigma(\eta) \in \mathbb{G}(a, b)\right\} .
$$

$$
\begin{aligned}
& \mathbb{G}(a, b)=\mathbb{G}^{\prime}(a, b), \quad \# \dot{\mathfrak{U}}\left(\gamma^{(0)}, a, b\right)=O\left(b(a+b)^{s-2}\right) \text { for } \gamma^{(0)} \in \mathbb{F}_{n}, \\
& \# \mathbb{G}(a, b)=O\left(n^{1 / 2} b(a+b)^{s-2}\right), \\
& \sum_{\gamma \in \mathbb{F}_{n}} \frac{1}{|\operatorname{Nm}(\gamma)|}=O(\ln n) \quad \text { and } \quad \sum_{\gamma \in \mathbb{F}_{n}} \frac{1}{\mathrm{Nm}^{2}(\gamma)}=O(1) .
\end{aligned}
$$


Proof. It is easy to see that $\mathbb{G}(a, b) \supseteq \mathbb{G}^{\prime}(a, b)$. Let $\gamma \in \mathbb{G}(a, b)$. By Lemma 2. there exists $\eta \in \mathfrak{U}_{M \perp}$ with $\gamma \cdot \sigma(\eta) \in \mathbb{F}_{n}^{\prime}$. From (2.2.6), we obtain that there exists $\eta_{1} \in \mathfrak{U}_{M \perp}$ with $\gamma^{(1)}=\gamma \cdot \sigma\left(\eta \eta_{1}\right) \in \mathbb{F}_{n}$. By (2.2.9) and (2.2.10), we get that $\gamma=\gamma^{(1)} \sigma\left(\left(\eta \eta_{1}\right)^{-1}\right) \in \mathbb{G}^{\prime}(a, b)$ and $\mathbb{G}(a, b)=\mathbb{G}^{\prime}(a, b)$. Let

$\mathbf{m}=\left(m_{1}, \ldots, m_{s}\right) \in \mathbb{Z}^{s}, m_{1}+\cdots+m_{s}=0, \boldsymbol{\kappa}=\left(\kappa_{1}, \ldots, \kappa_{s}\right), \kappa_{i} \in\{-1,1\}(i=$ $1, \ldots, s), \nu(\mu)=s$ if $\mu \neq s, \nu(\mu)=1$ if $\mu=s, j \geq 0$ and

$$
\begin{aligned}
& B(\mathbf{m}, \mu, \boldsymbol{\kappa}, j) \\
& =\prod_{1 \leq i<\nu(\mu)}\left(\kappa_{i} 2^{m_{i}}, \kappa_{i} 2^{m_{i}+1}\right] \times\left(j \kappa_{\nu(\mu)} 2^{-m_{\nu(\mu)}} C_{M}^{s},(j+1) \kappa_{\nu(\mu)} 2^{-m_{\nu(\mu)}} C_{M}^{s}\right] \\
& \prod_{\nu(\mu)<i \leq s}\left(\kappa_{i} 2^{m_{i}}, \kappa_{i} 2^{m_{i}+1}\right] .
\end{aligned}
$$

It is easy to see that

$$
B\left(\mathbf{m}_{1}, \mu, \boldsymbol{\kappa}_{1}, j_{1}\right) \cap B\left(\mathbf{m}_{2}, \mu, \boldsymbol{\kappa}_{2}, j_{2}\right)=\emptyset \quad \text { for } \quad\left(\mathbf{m}_{1}, \mu, \boldsymbol{\kappa}_{1}, j_{1}\right) \neq\left(\mathbf{m}_{2}, \mu, \boldsymbol{\kappa}_{2}, j_{2}\right) .
$$

Applying (1.4), we have for every $\mu \in[1, s]$ that

$$
\Gamma^{\perp} \backslash\{0\}=\bigcup_{\kappa_{1}, \ldots, \kappa_{s} \in\{-1,1\}} \bigcup_{\substack{\mathbf{m} \in \mathbb{Z}^{s}, m_{1}+\cdots+m_{s}=0}} \bigcup_{j \geq 0} \bigcup_{\boldsymbol{\gamma} \in B(\mathbf{m}, \mu, \boldsymbol{\kappa}, j)} \gamma .
$$

Let

$$
\gamma^{(1)}, \gamma^{(2)} \in \Gamma^{\perp} \cap B(\mathbf{m}, \mu, \boldsymbol{\kappa}, j) .
$$

From (2.2.13), we see that

$$
\left|\mathrm{Nm}\left(\gamma^{(1)}-\gamma^{(2)}\right)\right|<C_{M}^{s}
$$

By (1.4), we obtain that $\gamma^{(1)}=\gamma^{(2)}$ and

$$
\# \Gamma^{\perp} \cap B(\mathbf{m}, \mu, \boldsymbol{\kappa}, j) \leq 1 \text {. }
$$

Suppose

$$
\eta \in \mathfrak{U}_{M \perp} \cap B(\mathbf{m}, \mu, \boldsymbol{\kappa}, j) .
$$

Using (2.2.13), we have that

$$
1=|\operatorname{Nm}(\eta)|=\left(j+z_{1}\right) C_{M}^{s} 2^{z_{2}(s-1)} \quad \text { with } \quad z_{1}, z_{2} \in[0,1] .
$$

Hence

$$
-1+2^{1-s} / C_{M}^{s} \leq j \leq 1 / C_{M}^{s} .
$$

Applying (2.2.16), we get

$$
\sum_{j \geq 0} \# \mathfrak{U}_{M^{\perp}} \cap B(m, \mu, \kappa, j) \leq 2+1 / C_{M}^{s} .
$$




\section{GAUSSIAN LIMITING DISTRIBUTION OF LATTICE POINTS IN PARALLEPIDED}

We denote $\sigma^{-1}(B(m, \mu, \boldsymbol{\kappa}, j))$ and $\sigma^{-1}\left(\dot{\mathcal{U}}\left(\boldsymbol{\gamma}^{(0)}, a, b\right)\right)$ by the same symbols $B(m, \mu, \boldsymbol{\kappa}, j)$ and $\dot{\mathfrak{U}}\left(\boldsymbol{\gamma}^{(0)}, a, b\right)$. Now let

$$
\ddot{\mathfrak{U}}_{\mu}\left(\gamma^{(0)}, a, b\right)=\left\{\gamma \in \dot{\mathfrak{U}}\left(\gamma^{(0)}, a, b\right) \quad: \quad\left|\gamma_{i}\right| \leq\left|\gamma_{\mu}^{(0)}\right|, \quad i=1, \ldots, s\right\} .
$$

It is easy to see that

Let

$$
\dot{\mathfrak{U}}\left(\gamma^{(0)}, a, b\right)=\bigcup_{\mu \in[1, s]} \ddot{\mathfrak{U}}_{\mu}\left(\gamma^{(0)}, a, b\right) .
$$

$$
\eta \in \ddot{\mathfrak{U}}_{\mu}\left(\gamma^{(0)}, a, b\right) \cap B(m, \mu, \boldsymbol{\kappa}, j) .
$$

Denote $m_{i} \in \mathbb{Z}(i=1, \ldots, s)$ from the following condition:

$$
\log _{2}\left|\sigma(\eta)_{i}\right|=m_{i}+z_{i} \quad \text { with } \quad z_{i} \in[0,1) .
$$

By (2.2.8) and (2.2.20), we obtain

$$
\log _{2}\left|\left(\gamma^{(0)} \sigma(\eta)\right)_{\mu}\right|=\log _{2}\left|\gamma_{\mu}^{(0)}\right|+m_{\mu}+z_{\mu} \in(a, a+b]
$$

and

$$
m_{\mu} \in J_{1}:=\left(a-1-\log _{2}\left|\gamma_{\mu}^{(0)}\right|, a+b-\log _{2}\left|\gamma_{\mu}^{(0)}\right|\right] \cap \mathbb{Z} \quad \text { with } \quad \# J_{1} \leq b+2 .
$$

From (2.2.8), (2.2.20) and (1.4), we get

$\log _{2}\left|\gamma_{i}^{(0)} \sigma(\eta)_{i}\right|=\log _{2}\left|\gamma_{i}^{(0)}\right|+m_{i}+z_{i} \leq a+b \quad$ and $\quad m_{i} \leq a+b-\log _{2}\left|\gamma_{i}^{(0)}\right|$.

Using (2.2.23), (2.2.21) and (2.2.21), we derive that

$$
\begin{aligned}
\log _{2}\left|\gamma_{i}^{(0)} \sigma(\eta)_{i}\right| & =\log _{2}\left|\gamma_{i}^{(0)}\right|-\sum_{j \in[1, s], j \neq i} \log _{2}\left|\sigma(\eta)_{i}\right| \\
& \geq \log _{2}\left|\gamma_{i}^{(0)}\right|-\sum_{j \in[1, s], j \neq i}\left(m_{j}+1\right) \\
& \geq \sum_{j \in[1, s]} \log _{2}\left|\gamma_{j}^{(0)}\right|-(s-1)(a+b+1) \\
& =-(s-1)(a+b+1)+\log _{2}\left|\operatorname{Nm}\left(\gamma^{(0)}\right)\right| \\
& \geq-(s-1)(a+b+1)+\log _{2} C_{M}^{s} .
\end{aligned}
$$

By (2.2.23), we have $m_{i} \in\left[\log _{2}\left|\gamma_{i}^{(0)} \sigma(\eta)_{i}\right|-\log _{2}\left|\gamma_{i}^{(0)}\right|-1, a+b-\log _{2}\left|\gamma_{i}^{(0)}\right|\right]$. Hence

$$
\begin{aligned}
m_{i} \in J_{2}:=\left[-1-(s-1)(a+b+1)+s \log _{2} C_{M}\right. & \\
& \left.\quad-\log _{2}\left|\gamma_{i}^{(0)}\right|, a+b-\log _{2}\left|\gamma_{i}^{(0)}\right|\right],
\end{aligned}
$$

with $\# J_{2} \leq s(a+b+1)+2+s\left|\log _{2} C_{M}\right|$. 
We fix $\mu \in[1, s]$ and we consider (2.2.15). For given $m_{1}, \ldots, m_{\nu(\mu)-1}$, $m_{\nu(\mu)+1}, \ldots, m_{s}$, we take $m_{\nu(\mu)}=-\sum_{i \in[1, s], i \neq \nu(\mu)} m_{i}$. By (2.2.15), we get

$$
\begin{aligned}
& \# \ddot{\mathfrak{U}}_{\mu}\left(\gamma^{(0)}, a, b\right) \\
& \leq \sum_{\kappa_{1}, \ldots, \kappa_{s} \in\{-1,1\}} \sum_{m_{\mu} \in J_{1}} \sum_{\substack{m_{i} \in J_{2} \\
i \neq \mu, \nu(\mu)}} \sum_{j \geq 0} \#\left(\dot{\mathfrak{U}}\left(\gamma^{(0)}, a, b\right) \cap B(m, \mu, \boldsymbol{\kappa}, j)\right) .
\end{aligned}
$$

Bearing in mind (2.2.18), 2.2.19) and (2.2.22), we obtain

$$
\# \dot{\mathfrak{U}}\left(\gamma^{(0)}, a, b\right)=O\left(\# J_{1}\left(\# J_{2}\right)^{s-2}\right)=O\left(b(a+b)^{s-2}\right) .
$$

Hence, the assertion (2.2.11) is proved.

Let $F_{1} \subset \mathbb{R}^{s}$ be a fundamental domain for the field $\mathcal{K}$, and let

$F_{2}=\left\{\gamma \in \Gamma^{\perp} \backslash\{0\}:\left|\gamma_{i}\right||\operatorname{Nm}(\gamma)|^{-1 / s} \in\left[1 / c_{0}, c_{0}\right], i=1, \ldots, s\right\} \quad$ (see (2.2.2) $)$.

By BS, pp. 312, 322], the points of $F_{1}$ can be arranged in a sequence $\dot{\gamma}^{(k)}$ so that $0<\left|\mathrm{Nm}\left(\dot{\gamma}^{(1)}\right)\right| \leq\left|\mathrm{Nm}\left(\dot{\gamma}^{(2)}\right)\right| \leq \cdots$ and $c^{(1)} k \leq\left|\mathrm{Nm}\left(\dot{\gamma}^{(k)}\right)\right| \leq c^{(2)} k$, $k=1,2, \ldots$ for some $c^{(2)}>c^{(1)}>0$. Therefore, the points of $F_{2}$ can be arranged in a sequence $\gamma^{(k)}$ so that

$$
0<\left|\operatorname{Nm}\left(\gamma^{(1)}\right)\right| \leq\left|\operatorname{Nm}\left(\gamma^{(2)}\right)\right| \leq \cdots
$$

and

$$
c^{(3)} k \leq\left|\operatorname{Nm}\left(\gamma^{(k)}\right)\right| \leq c^{(4)} k, \quad k=1,2, \ldots \quad \text { for some } \quad c^{(4)}>c^{(3)}>0 .
$$

Using (2.2.6), we have that

$$
\sum_{\gamma \in \mathbb{F}_{n}} 1 /|\operatorname{Nm}(\gamma)|=O(\ln (n)), \quad \sum_{\gamma \in \mathbb{F}_{n}} 1 / \mathrm{Nm}^{2}(\gamma)=O(1), \quad \text { and } \quad \# \mathbb{F}_{n}=O\left(n^{1 / 2}\right)
$$

By (2.2.9) and (2.2.24), we obtain

$$
\# \mathbb{G}(a, b) \leq \sum_{\gamma^{(0)} \in \mathbb{F}_{n}} \# \dot{\mathfrak{U}}\left(\gamma^{(0)}, a, b\right)=O\left(n^{1 / 2} b(a+b)^{s-2}\right) .
$$

Hence, Lemma 3 is proved.

\subsection{Diophantine inequalities}

We consider the following simple variant of the $\boldsymbol{S}$-unit theorem (see ESS, Theorem 1.1, p. 808]): Let $\beta_{1}, \ldots, \beta_{d} \in \mathcal{K}, \beta_{i} \neq 0, i=1, \ldots, d, \operatorname{deg}(\mathcal{K})=s$. We consider the equation

$$
\beta_{1} \eta_{1}+\cdots+\beta_{d} \eta_{d}=1 \text { with } \boldsymbol{\eta}=\left(\eta_{1}, \ldots, \eta_{d}\right) \in\left(\mathfrak{U}_{M^{\perp}}\right)^{d} .
$$

A solution $\boldsymbol{\eta}$ of (2.3.1) is called non-degenerate if $\sum_{i \in I} \beta_{i} \eta_{i} \neq 0$ for every nonempty subset $I$ of $\{1, \ldots, d\}$. 
TheOREM A. The number $A\left(\beta_{1}, \ldots, \beta_{d}\right)$ of non-degenerate solutions $\boldsymbol{\eta} \in\left(\mathfrak{U}_{M^{\perp}}\right)^{d}$ of equation (2.3.1) satisfies the estimate

$$
A\left(\beta_{1}, \ldots, \beta_{d}\right) \leq \exp \left((6 d)^{3 d} s\right) .
$$

Linear forms in logarithms. Write $\Lambda$ for the linear form in logarithms,

$$
\Lambda=b_{1} \log \alpha_{1}+\cdots+b_{k} \log \alpha_{k},
$$

where $b_{1}, \ldots, b_{k}$ are integers, $\left|b_{i}\right| \leq B(i=1, \ldots, k), B \geq e$. We shall assume that $\alpha_{1}, \ldots, \alpha_{k}$ are non-zero algebraic numbers with heights at most $A_{1}, \ldots, A_{k}$ $($ all $\geq e)$, respectively.

Theorem B. BW, Theorem 2.15, p. 42] If $\Lambda \neq 0$, then

$$
|\Lambda|>\exp \left(-(16 k d)^{2(k+2)} \ln A_{1} \cdots \ln A_{k} \ln B\right),
$$

where $d$ denote the degrees of $\mathbb{Q}\left(\alpha_{1}, \ldots, \alpha_{k}\right)$.

Let

$$
\begin{aligned}
& G^{(1)}=\left\{\gamma \in \Gamma^{\perp}:|\gamma| \leq N,|\operatorname{Nm}(\gamma)| \leq n^{1 / 2}\right. \text { and } \\
& G^{(2)}\left.=\left\{\gamma \in \Gamma^{\perp}:|\gamma|>N^{5}\right\}, \quad\left|N_{i} \gamma_{i}\right|>2^{(\ln n)^{4}} \forall i \in[1, s]\right\}, \\
& G^{(3)}=\left\{\gamma \in \Gamma^{\perp}:|\gamma| \leq N^{5},|\operatorname{Nm}(\gamma)|>n^{1 / 2}\right\}, \\
& G^{(4)}=\left\{\gamma \in \Gamma^{\perp}: N<|\gamma| \leq N^{5},|\operatorname{Nm}(\gamma)| \leq n^{1 / 2}\right\} \quad \text { and } \\
& G^{(5)}=\left\{\gamma \in \Gamma^{\perp} \backslash\{0\} \quad:|\gamma| \leq N,|\operatorname{Nm}(\gamma)| \leq n^{1 / 2} \quad\right. \text { and } \\
&\left.\exists i \in[1, s] \quad \text { with } \quad\left|N_{i} \gamma_{i}\right| \leq 2^{(\ln n)^{4}}\right\}
\end{aligned}
$$

It is easy to see that $G^{(i)} \cap G^{(j)}=\emptyset$ for $i \neq j$, and

Let

$$
\Gamma^{\perp} \backslash\{0\}=G^{(1)} \cup G^{(2)} \cup G^{(3)} \cup G^{(4)} \cup G^{(5)} .
$$

$$
\begin{aligned}
& \dot{G}_{0}=\left\{\gamma \in G^{(1)}: \max _{1 \leq j \leq s}\left|\gamma_{i}\right| \leq 2^{n^{4 / 9}}\right\}, \\
& \dot{G}_{i}=\left\{\gamma \in G^{(1)}: \max _{1 \leq j \leq s}\left|\gamma_{i}\right| \in\left(2^{i n^{4 / 9}}, 2^{(i+1) n^{4 / 9}-n^{2 / 9}}\right]\right\},
\end{aligned}
$$

and

$$
\ddot{G}_{i}=\left\{\gamma \in G^{(1)}: \max _{1 \leq j \leq s}\left|\gamma_{i}\right| \in\left(2^{(i+1) n^{4 / 9}-n^{2 / 9}}, 2^{(i+1) n^{4 / 9}}\right]\right\}, \quad i=1,2, \ldots
$$


By (2.3.4) and (2.3.8), we have that $\dot{G}_{i} \cap \dot{G}_{j}=\emptyset$ for $i \neq j, \dot{G}_{i} \cap \ddot{G}_{j}=\emptyset$ and

$$
G^{(1)}=\dot{G}_{0} \cup \bigcup_{i=1}^{\left[n^{5 / 9}\right]}\left(\dot{G}_{i} \cup \ddot{G}_{i}\right) .
$$

Lemma 4. There exist $\dot{c}, \ddot{c}>0$ such that for all $\nu \in[1, s]$ and $\kappa \in\{-1,1\}$

$$
\min _{\boldsymbol{\gamma}^{(1)}, \boldsymbol{\gamma}^{(2)} \in G^{(1)}, \gamma_{\nu}^{(1)} \neq \kappa \gamma_{\nu}^{(2)}} N_{\nu}\left|\gamma_{\nu}^{(1)}-\kappa \gamma_{\nu}^{(2)}\right| \geq N_{\nu}\left|\gamma_{\nu}^{(2)}\right| \exp \left(-\ddot{c}(\ln n)^{3}\right) \geq \dot{c} n^{20 s} .
$$

Proof. Let $\gamma_{\nu}^{(1)} / \gamma_{\nu}^{(2)} \kappa<0$. From (2.3.4), we obtain

$$
\min _{\gamma^{(1)}, \gamma^{(2)} \in G^{(1)}, \gamma_{\nu}^{(1)} \neq \kappa \gamma_{\nu}^{(2)}} N_{\nu}\left|\gamma_{\nu}^{(1)}-\kappa \gamma_{\nu}^{(2)}\right| \geq N_{\nu}\left|\gamma_{\nu}^{(2)}\right| \geq 2^{(\ln n)^{4}}
$$

Now let $\gamma_{\nu}^{(1)} / \gamma_{\nu}^{(2)} \kappa>0$. Taking into account that $|\exp (x)-1| \geq|x|$ any real $x$, we get

$$
\left|\gamma_{\nu}^{(1)}-\kappa \gamma_{\nu}^{(2)}\right|=\left|\gamma_{\nu}^{(2)}\left(\exp \left(\ln \left(\kappa \gamma_{\nu}^{(1)} / \gamma_{\nu}^{(2)}\right)\right)-1\right)\right| \geq\left|\gamma_{\nu}^{(2)} \ln \left(\kappa \gamma_{\nu}^{(1)} / \gamma_{\nu}^{(2)}\right)\right| .
$$

By (2.2.6), (2.2.8), (2.2.9) and (2.3.4), we have that there exists $\left(\dot{\gamma}_{\nu}^{(k)}, \eta_{k}\right)$ such that $\gamma_{\nu}^{(k)}=\dot{\gamma}_{\nu}^{(k)} \cdot \sigma_{\nu}\left(\eta_{k}\right)$, where $\dot{\gamma}^{(k)} \in \mathbb{F}_{n}$ and $\eta_{k}$ is a unit in $\mathcal{K}(k=1,2)$. Let $\dot{\gamma}^{(k)}=\sigma\left(\mathfrak{f}^{(k)}\right)$ with some $\mathfrak{f}^{(k)} \in M^{\perp}(k=1,2)$. Using (2.2.1), (2.2.6) and (2.3.4), we obtain

$$
\begin{aligned}
\gamma_{\nu}^{(k)}=\sigma_{\nu}\left(\mathfrak{f}^{(k)}\right)(-1)^{a^{(k)}} \sigma_{\nu}\left(\eta_{2,1}\right)^{a_{1}^{(k)}} \cdots \sigma_{\nu}\left(\eta_{2, s-1}\right)^{a_{s-1}^{(k)}} & \\
\left|\sigma_{i}\left(\mathfrak{f}^{(k)}\right)\right| & \leq c_{0} n^{1 /(2 s)} \quad \text { for } \quad i=1, \ldots, s,
\end{aligned}
$$

and

$$
\begin{aligned}
\mid a_{1}^{(k)} \ln \left(\sigma_{\nu}\left(\eta_{2,1}\right)\right) & +\cdots+a_{s-1}^{(k)} \ln \left(\sigma_{\nu}\left(\eta_{2, s-1}\right)\right) \mid \\
& \leq|\ln | \gamma_{\nu}^{(k)}||+|\ln | \sigma_{\nu}\left(\mathfrak{f}^{(k)}\right)|| \leq \ln N+1 /(2 s) \ln n+\ln \left(c_{0}\right) .
\end{aligned}
$$

Bearing in mind that $\operatorname{det}\left(\left(\ln \left(\sigma_{i}\left(\eta_{2, j}\right)\right)\right)_{1 \leq i, j \leq s-1}\right) \neq 0$ (see [BS, pp. 104, 115]), we get that there exists $\tilde{C}_{1}>0$ such that

$$
\left|a_{i}^{(k)}\right|<\tilde{C}_{1} n \quad \text { for } \quad i=1, \ldots, s-1, k=1,2 \quad \text { and } \quad n=\left[\log _{2} N\right]+1 .
$$

Let $\kappa_{1}=\operatorname{sign}\left(\gamma_{\nu}^{(1)} / \gamma_{\nu}^{(2)}\right)$, where $\operatorname{sign}(x)=1$ for $x>0$ and $\operatorname{sign}(x)=-1$ for $x<0$. We see that

$$
\begin{aligned}
\ln \left|\gamma_{\nu}^{(1)} / \gamma_{\nu}^{(2)}\right|= & \ln \left(\kappa_{1} \gamma_{\nu}^{(1)} / \gamma_{\nu}^{(2)}\right)=\ln \left(\kappa_{1}(-1)^{a^{(1)}} \sigma_{\nu}\left(\mathfrak{f}^{(1)}\right)\right)-\ln \left((-1)^{a^{(2)}} \sigma_{\nu}\left(\mathfrak{f}^{(2)}\right)\right) \\
& +\left(a_{1}^{(1)}-a_{1}^{(2)}\right) \ln \left(\sigma_{\nu}\left(\eta_{2,1}\right)\right)+\cdots+\left(a_{s-1}^{(1)}-a_{s-1}^{(2)}\right) \ln \left(\sigma_{\nu}\left(\eta_{2, s-1}\right)\right) .
\end{aligned}
$$




\section{GAUSSIAN LIMITING DISTRIBUTION OF LATTICE POINTS IN PARALLEPIDED}

Let $\tilde{C}_{2} \max _{i \in[1, s-1]} H\left(\eta_{2, i}\right)$, where $H(\alpha)$ is the height of $\alpha$. By (1.4), $C_{M}^{-1} \mathfrak{f}^{(k)}$ is an algebraic integer $(k=1,2)$. Thus

$$
f(x)=x^{s}+f_{s-1} x^{s-1}+\cdots+f_{0}=\left(x-\sigma_{1}\left(C_{M}^{-1} \mathfrak{f}^{(k)}\right)\right) \cdots\left(x-\sigma_{s}\left(C_{M}^{-1} \mathfrak{f}^{(k)}\right)\right)
$$

is the characteristic polynomial of $C_{M}^{-1} \mathfrak{f}^{(k)}$. Hence

$$
H\left(C_{M}^{-1} \mathfrak{f}^{(k)}\right) \leq \max _{i \in[0, s-1]}\left|f_{i}\right| .
$$

From (2.3.12), we have that

$$
H\left(C_{M}^{-1} \mathfrak{f}^{(k)}\right) \leq\left(2 C_{M}^{-1} c_{0} n^{1 /(2 s)}\right)^{s}
$$

and

$$
H\left(\mathfrak{f}^{(k)}\right) \leq\left(2 C_{M}^{-2} c_{0} n^{1 /(2 s)}\right)^{s} .
$$

Applying Theorem B with $d=s, k=s+1, \alpha_{1}=\kappa_{1}(-1)^{a^{(1)}} \sigma_{\nu}\left(\mathfrak{f}^{(1)}\right)$, $\alpha_{2}=(-1)^{a^{(2)}} \sigma_{\nu}\left(\mathfrak{f}^{(2)}\right), \alpha_{3}=\sigma_{\nu}\left(\eta_{2,1}\right), \ldots, \alpha_{s+1}=\sigma_{\nu}\left(\eta_{2, s-1}\right)$, $A_{1}=A_{2}=\left(2 C_{M}^{-2} c_{0} n^{1 /(2 s)}\right)^{s}, A_{3}=\cdots=A_{s-1}=\tilde{C}_{2}$ and $B=2 \tilde{C}_{1} n$, we obtain

$$
\left|\ln \left(\kappa_{1} \gamma_{\nu}^{(1)} / \gamma_{\nu}^{(2)}\right)\right| \geq \exp \left(-\ddot{c}_{\nu}(\ln n)^{3}\right),
$$

with some $\ddot{c}_{\nu}>0$. Taking into account (2.3.11) and that $N_{\nu}\left|\gamma_{\nu}^{(2)}\right| \geq 2^{(\ln n)^{4}}$, we have

$$
\min _{\gamma^{(1)}, \gamma^{(2)} \in G^{(1)}, \gamma_{\nu}^{(1)} \neq \kappa \gamma_{\nu}^{(2)}} N_{\nu}\left|\gamma_{\nu}^{(1)}-\kappa \gamma_{\nu}^{(2)}\right| \geq N_{\nu}\left|\gamma_{\nu}^{(2)}\right| \exp \left(-\ddot{c}_{\nu}(\ln n)^{3}\right) \geq \dot{c}_{\nu} n^{20 s},
$$

with some $\dot{c}_{\nu}>0$. Now using (2.3.10), we get the assertion of Lemma 4,

\subsection{Upper bound of the variance of $\mathcal{R}\left(\boldsymbol{\theta} \cdot \mathbf{N} \cdot \mathbb{K}^{s}+\mathrm{x}, \Gamma\right)$}

In this subsection, we prove that $\mathcal{A}\left(G_{1}\right)$ is the main part of $\dot{\mathcal{R}}$. In (2.3.9), we decomposed $G_{1}$ to essential parts $\left(\dot{G}_{i}\right)_{i \in[1, n]}$ and to auxiliary parts $\left(\ddot{G}_{i}\right)_{i \in[1, n]}$. This allows us to obtain in Lemma 11 that the approximation of $\dot{\mathcal{R}}$ be the sum of essential elements $\left(\mathcal{A}\left(\dot{G}_{i}\right)\right)$. The random variables $\left(\mathcal{A}\left(\dot{G}_{i}\right)\right)$ are almost independents. Thus in Lemma 11 we do the main step to construct the martingale approximation for $\dot{\mathcal{R}}$.

Let

$$
\begin{aligned}
& \mathcal{A}(G)=(\operatorname{det} \Gamma)^{-1} \sum_{\gamma \in G} \widehat{\mathbb{1}}_{\mathcal{O}_{\boldsymbol{\theta} \cdot \mathbf{N}}}(\gamma) \hat{\omega}(\tau \gamma) e(\langle\boldsymbol{\gamma}, \mathbf{x}\rangle), \\
& \tilde{\mathcal{A}}(G)=(\operatorname{det} \Gamma)^{-1} \sum_{\gamma \in G}\left|\widehat{\mathbb{1}}_{\mathcal{O}_{\boldsymbol{\theta} \cdot \mathbf{N}}}(\gamma) \hat{\omega}(\tau \gamma)\right|,
\end{aligned}
$$

and let 


$$
\mathcal{B}(G, \boldsymbol{\kappa})=\sum_{\gamma \in G} \frac{(\operatorname{det} \Gamma)^{-1} \hat{\omega}(\tau \gamma)}{(2 \pi \sqrt{-1})^{s} \operatorname{Nm}(\gamma)} e\left(\sum_{k=1}^{s} \gamma_{k}\left(\kappa_{k}\left(\theta_{k} N_{k}\right) / 2+x_{k}\right)\right) .
$$

We obtain from 2.1.2

$$
\mathcal{A}(G)=\sum_{\kappa_{1}, \ldots, \kappa_{s} \in\{-1,1\}} \kappa_{1} \kappa_{2} \cdots \kappa_{s} \mathcal{B}(G, \kappa) .
$$

Using the Cauchy-Schwartz inequality, we get

$$
|\mathcal{A}(G)|^{2} \leq 2^{s} \sum_{\kappa_{1}, \ldots, \kappa_{s} \in\{-1,1\}}|\mathcal{B}(G, \boldsymbol{\kappa})|^{2} .
$$

By (2.3.7) and (2.1.6), we see that

$$
\ddot{\mathcal{R}}\left(\mathcal{O}_{\boldsymbol{\theta} \cdot \mathbf{N}}+\mathbf{x}, \Gamma\right)=\mathcal{A}\left(G^{(1)}\right)+\cdots+\mathcal{A}\left(G^{(5)}\right) .
$$

Let

$$
h(\gamma)=\frac{(\operatorname{det} \Gamma)^{-1} \hat{\omega}(\tau \gamma)}{(2 \pi \sqrt{-1})^{s} \operatorname{Nm}(\gamma)} e\left(\sum_{k=1}^{s} \gamma_{k} x_{k}\right) .
$$

It is easy to see that

and

$$
\mathcal{B}(G, \boldsymbol{\kappa})=\sum_{\gamma \in G} h(\gamma) e\left(\sum_{k=1}^{s} \kappa_{k} \gamma_{k} \theta_{k} N_{k} / 2\right),
$$

$$
\mathcal{A}(G)=\sum_{\kappa_{1}, \ldots, \kappa_{s} \in\{-1,1\}} \kappa_{1} \kappa_{2} \cdots \kappa_{s} \sum_{\gamma \in G} h(\gamma) e\left(\sum_{k=1}^{s} \kappa_{k} \gamma_{k} \theta_{k} N_{k} / 2\right) .
$$

LEMma 5. With the notations as above

$$
\mathcal{A}\left(G^{(2)}\right)=O(1 / N) .
$$

Proof. By (2.4.3) and (2.1.5) we have that

$$
\left|\mathcal{B}\left(G^{(2)}, \boldsymbol{\kappa}\right)\right| \leq c_{2} \sum_{\gamma \in G^{(2)}} \frac{(\operatorname{det} \Gamma)^{-1}(\tau|\gamma|)^{-2 s}}{(2 \pi)^{s}|\operatorname{Nm}(\gamma)|}, \quad \tau=N^{-2}
$$

Notice that for every lattice $\mathfrak{L} \in \mathbb{R}^{s}$, one has the bound (see, e.g., GL, pp. 141-142])

Hence

$$
\#\{\gamma \in \mathfrak{L}: j \leq|\gamma| \leq j+1\}=O\left(j^{s-1}\right) .
$$

$$
\begin{aligned}
\sum_{\gamma \in \Gamma^{\perp}:|\gamma| \geq N^{5}}|\gamma|^{-2 s} & \leq \sum_{j \geq N^{5}} \sum_{\gamma \in \Gamma^{\perp}:|\gamma| \in[j, j+1)}|\gamma|^{-2 s} \\
& =O\left(\sum_{j \geq N^{5}} j^{-s-1}\right)=O\left(N^{-5 s}\right) .
\end{aligned}
$$


By (1.4), (2.3.5) and (2.4.10), we obtain

$$
\left|\mathcal{B}\left(G^{(2)}, \boldsymbol{\kappa}\right)\right| \leq c_{2}\left(C_{M}^{s} \operatorname{det} \Gamma(2 \pi)^{s}\right)^{-1} \sum_{\boldsymbol{\gamma} \in \Gamma^{\perp}:|\boldsymbol{\gamma}| \geq N^{5}} N^{4 s}|\boldsymbol{\gamma}|^{-2 s}=O\left(N^{-s}\right) .
$$

Using (2.4.4), we get the assertion of Lemma 5 .

Lemma 6. Let $\gamma^{(i)} \in \Gamma^{\perp}, i=1,2, \gamma^{(1)} \neq \gamma^{(2)}$. Then

$$
\left|\mathbf{E}\left[e\left(\left\langle\gamma^{(1)}-\gamma^{(2)}, \boldsymbol{\theta} \cdot \mathbf{N}\right\rangle / 2+\beta\right)\right]\right| \leq \frac{1}{\pi^{s} C_{M}^{s} N} .
$$

P r o of. Bearing in mind that

$$
\left|\int_{0}^{1} e(x z) d x\right|=\left|\frac{e(z)-1}{2 \pi z}\right| \leq \frac{1}{|\pi z|}, \quad \text { with } \quad z \neq 0
$$

(1.4) and that $N_{1} \cdots N_{s}=N$, we have

$$
\left|\mathbf{E}\left[e\left(\left\langle\gamma^{(1)}-\gamma^{(2)}, \boldsymbol{\theta} \cdot \mathbf{N}\right\rangle / 2+\beta\right)\right]\right| \leq \frac{1}{\pi^{s} N\left|\operatorname{Nm}\left(\gamma^{(1)}-\gamma^{(2)}\right)\right|} \leq \frac{1}{\pi^{s} C_{M}^{s} N} .
$$

LEMMA 7. With the notations as above

$$
\mathbf{E}\left[\left|\mathcal{A}\left(G^{(1)}\right)\right|^{2}\right]=O\left(n^{s-1}\right), \quad \mathbf{E}\left[\left|\mathcal{A}\left(G^{(3)}\right)\right|^{2}\right]=O\left(n^{s-3 / 2}\right),
$$

and

$$
|\mathcal{A}(\dot{G})| \leq \tilde{\mathcal{A}}(\dot{G})=O\left(n^{s}\right), \quad \text { with } \quad G \subseteq G^{(1)} .
$$

Proof. By (2.4.3), (2.4.5) and the Cauchy-Schwartz inequality, we obtain that

$$
\begin{aligned}
& \mathbf{E}\left[|\mathcal{A}(G)|^{2}\right] \leq 2^{s} \sum_{\substack{\kappa_{1}, \ldots, \kappa_{s} \in\{-1,1\}\\
}}\left(\sum_{\boldsymbol{\gamma} \in G} \frac{(\operatorname{det} \Gamma)^{-2}|\hat{\omega}(\tau \gamma)|^{2}}{(2 \pi)^{2 s}|\operatorname{Nm}(\boldsymbol{\gamma})|^{2}}\right. \\
&+\sum_{\substack{\boldsymbol{\gamma}^{(1)}, \boldsymbol{\gamma}^{(2)} \in G \\
\boldsymbol{\gamma}^{(1)} \neq \boldsymbol{\gamma}^{(2)}}} \frac{(\operatorname{det} \Gamma)^{-2}\left|\hat{\omega}\left(\tau \gamma^{(1)}\right)\right|\left|\hat{\omega}\left(\tau \gamma^{(2)}\right)\right|}{(2 \pi)^{2 s}\left|\operatorname{Nm}\left(\boldsymbol{\gamma}^{(1)}\right)\right|\left|\mathrm{Nm}\left(\boldsymbol{\gamma}^{(2)}\right)\right|} \\
&\left.\times\left|\mathbf{E}\left[e\left(\left\langle\boldsymbol{\gamma}^{(1)}-\boldsymbol{\gamma}^{(2)}, \boldsymbol{\kappa} \cdot \boldsymbol{\theta} \cdot N\right\rangle / 2+\beta\right)\right]\right|\right)
\end{aligned}
$$

with $\beta=\sum_{k=1}^{s}\left(\gamma_{k}^{(1)}-\gamma_{k}^{(2)}\right) x_{k}$. 
Applying Lemma 6, we get

$$
\mathbf{E}\left[|\mathcal{A}(G)|^{2}\right] \leq 2^{2 s} \sum_{\mu \in[1, s]} S_{1, \mu}(G)+\frac{2^{2 s}}{\pi^{s} C_{M}^{s} N} S_{2}(G, G)
$$

with

$$
S_{1, \mu}(G)=\sum_{\gamma \in G,\left|\gamma_{i}\right| \leq\left|\gamma_{\mu}\right|, i=1, \ldots, s} \frac{(\operatorname{det} \Gamma)^{-2}|\hat{\omega}(\tau \gamma)|^{2}}{(2 \pi)^{2 s}|\operatorname{Nm}(\gamma)|^{2}}
$$

and

$$
S_{2}(\dot{G}, \ddot{G})=\sum_{\gamma^{(1)} \in \dot{G}, \gamma^{(2)} \in \ddot{G},} \gamma^{(1) \neq \gamma^{(2)}} \frac{(\operatorname{det} \Gamma)^{-2}\left|\hat{\omega}\left(\tau \gamma^{(1)}\right)\right|\left|\hat{\omega}\left(\tau \gamma^{(2)}\right)\right|}{(2 \pi)^{2 s}\left|\operatorname{Nm}\left(\gamma^{(1)}\right)\right|\left|\operatorname{Nm}\left(\gamma^{(2)}\right)\right|} .
$$

We fix $\mu \in[1, s]$, and we consider $S_{1, \mu}(G)$. Let $\gamma \in B(m, \mu, \boldsymbol{\kappa}, j)$. According to (2.2.13) and (2.2.16), we have

$$
|\operatorname{Nm}(\boldsymbol{\gamma})|=\left(j+z_{1}\right) C_{M}^{s} 2^{z_{2}(s-1)}, \quad \text { and } \quad \# \Gamma^{\perp} \cap B(\mathbf{m}, \mu, \boldsymbol{\kappa}, j) \leq 1
$$

with $z_{1}, z_{2} \in[0,1]$. By (2.3.5) and (2.4.17), we obtain

$$
j+1 \geq n^{1 / 2}\left(C_{M}^{s} 2^{s-1}\right)^{-1} \quad \text { for } \quad \gamma \in G^{(3)} .
$$

Hence

$$
\begin{gathered}
\sum_{j \geq 0} \sum_{\gamma \in G^{(3)} \cap B(m, \mu, \kappa, j)} \frac{1}{|\operatorname{Nm}(\gamma)|^{2}}=O\left(\sum_{j \geq n^{1 / 2}} 1 / j^{2}\right)=O\left(n^{-1 / 2}\right) \\
\sum_{j \geq 0} \sum_{\gamma \in G \cap B(m, \mu, \boldsymbol{\kappa}, j)} \frac{1}{|\operatorname{Nm}(\gamma)|^{2}}=O\left(\sum_{j \geq 1} 1 / j^{2}\right)=O(1), \text { for } G \subseteq G^{(1)}
\end{gathered}
$$

Bearing in mind that $\mathrm{Nm}(\gamma) \leq N^{5 s}$ for $\gamma \in G^{(1)} \cup G^{(3)}$ and $n=\left[\log _{2} N\right]+1$, we get from (2.3.5) and (2.4.17) that

$$
\sum_{j \geq 0} \sum_{\gamma \in\left(G^{(1)} \cup G^{(3)}\right) \cap B(m, \mu, \kappa, j)} \frac{1}{|\mathrm{Nm}(\boldsymbol{\gamma})|}=O\left(\sum_{1 \leq j \leq N^{5 s}} 1 / j\right)=O(n) .
$$

By (2.2.13), (2.3.5) and (1.4), we have for $\gamma \in\left(G^{(1)} \cup G^{(3)}\right) \cap B(m, \mu, \boldsymbol{\kappa}, j)$ that

$$
\log _{2}\left|\gamma_{i}\right| \in\left[m_{i}, m_{i}+1\right), \quad i \in[1, s], i \neq \nu(\mu), \quad\left|\gamma_{i}\right| \leq N^{5}, \quad|\operatorname{Nm}(\gamma)| \geq C_{M}^{s},
$$

$C_{M}^{s} N^{-5(s-1)} \leq\left|\gamma_{i}\right|, \quad s \log _{2} C_{M}-5(s-1) \log _{2} N \leq \log _{2}\left|\gamma_{i}\right| \leq 5 \log _{2} N, i \in[1, s]$.

Therefore

$$
m_{i} \in J, \quad i \in[1, s], i \neq \nu(\mu) \quad \text { with } \quad J=\left[s \log _{2} C_{M}-5(s-1) n, 5 n\right] .
$$


From (2.2.15), (2.1.5) and (2.4.15), we derive

$$
S_{1, \mu}(G) \leq \sum_{\kappa_{1}, \ldots, \kappa_{s} \in\{-1,1\}} \sum_{\substack{m_{1}+\ldots+m_{s}=0 \\ \mathbf{m} \in \mathbb{Z}^{s}, j \geq 0}} \sum_{\substack{\boldsymbol{\gamma} \in G \cap B\left(\mathbf{m}, \mu, \boldsymbol{\kappa}, j \\\left|\gamma_{i}\right| \leq\left|\gamma_{\mu}\right|, i=1, \ldots, s\right.}} \frac{(\operatorname{det} \Gamma)^{-2} c_{2}^{2}}{(2 \pi)^{2 s}|\operatorname{Nm}(\gamma)|^{2}}
$$

Hence, we obtain for $i=1,3$ that

$$
S_{1, \mu}\left(G^{(i)}\right) \leq \sum_{\kappa_{1}, \ldots, \kappa_{s} \in\{-1,1\}} \sum_{\substack{m_{k} \in J, k \in[1, s], k \neq \nu(\mu) \\ m_{1}+\ldots+m_{s}=0, j \geq 0}} \sum_{\boldsymbol{\gamma} \in G^{(i)} \cap B(\mathbf{m}, \mu, \boldsymbol{\kappa}, j)} \frac{(\operatorname{det} \Gamma)^{-2} c_{2}^{2}}{(2 \pi)^{2 s}|\operatorname{Nm}(\boldsymbol{\gamma})|^{2}}
$$

Applying (2.4.18), (2.4.19) and (2.4.21), we get that

$$
S_{1, \mu}\left(G^{(1)}\right)=O\left(n^{s-1}\right), \quad \text { and } \quad S_{1, \mu}\left(G^{(3)}\right)=O\left(n^{s-1-1 / 2}\right) .
$$

Analogously, we have from (2.4.16) and 2.4.20) that for $\dot{G}, \ddot{G} \subset G^{(1)} \cup G^{(3)}$

$$
S_{2}(\dot{G}, \ddot{G})=O\left(\left(\sum_{\kappa_{1}, \ldots, \kappa_{s} \in\{-1,1\}} \sum_{\substack{m_{k} \in J, k \in[1, s] \\ k \neq \nu(\mu), m_{1}+\ldots+m_{s}=0}} \sum_{j \geq 0} \sum_{\boldsymbol{\gamma} \in G_{0}} \frac{1}{\operatorname{Nm}(\gamma)}\right)^{2}\right)=O\left(n^{2 s}\right) \text {, }
$$

where $G_{0}=G^{(1)} \cup G^{(3)} \cap B(\mathbf{m}, \mu, \boldsymbol{\kappa}, j)$.

According to (2.4.14), we obtain (2.4.12). By (2.4.2) and (2.1.2), we have that

$$
\tilde{\mathcal{A}}(G) \leq(\operatorname{det} \Gamma)^{-1} c_{2} \sum_{\gamma \in G} 1 /|\operatorname{Nm}(\gamma)|
$$

Now using (2.4.20), similarly to (2.4.22)-(2.4.23), we get (2.4.13).

Hence, Lemma 7 is proved.

LEMMA 8. With the notations as above

$$
S_{1, \mu}(\tilde{G})=O\left(n^{s-11 / 9}\right), \quad \text { with } \quad \tilde{G}=\dot{G}_{0} \bigcup_{1 \leq i \leq n^{5 / 9}+1} \ddot{G}_{i}, \quad \mu=1, \ldots, s .
$$

Proof. Let $\gamma \in \ddot{G}_{i} \cap B(\mathbf{m}, \mu, \boldsymbol{\kappa}, j)$. By (2.2.13), we have that $\log _{2}\left|\gamma_{k}\right| \in$ $\left[m_{k}, m_{k}+1\right)$ with $k \in[1, s], k \neq \nu(\mu)$. From (2.3.8) and (1.4), we derive for $\left|\gamma_{i}\right| \leq\left|\gamma_{\mu}\right|, i=1, \ldots, s$ that

$$
\log _{2}\left|\gamma_{k}\right| \leq(i+1) n^{4 / 9}, \quad \log _{2}\left|\gamma_{k}\right| \geq s \log _{2} C_{M}-\sum_{j \in[1, s], j \neq k} \log _{2}\left|\gamma_{j}\right|, k=1, \ldots, s,
$$

and

$$
\log _{2}\left|\gamma_{\mu}\right|>(i+1) n^{4 / 9}-n^{2 / 9}
$$

Therefore

$m_{\mu} \in J_{1} \quad$ with $\quad J_{1}=\left((i+1) n^{4 / 9}-n^{2 / 9}-1,(i+1) n^{4 / 9}\right], \quad \# J_{1} \leq n^{2 / 9}+2$, 
and

$$
\begin{aligned}
m_{k} \in J_{2} \quad \text { with } \quad J_{2} & =\left(-(s-1)(i+1) n^{4 / 9}+s \log _{2} C_{M}-1,(i+1) n^{4 / 9}\right], \\
k & \in[1, s], k \neq \nu(\mu), \mu, \# J_{2}=O\left((i+1) n^{4 / 9}\right), i=1,2, \ldots
\end{aligned}
$$

By (2.4.22), we get that

$$
S_{1}\left(\ddot{G}_{i}\right) \leq \sum_{\kappa_{1}, \ldots, \kappa_{s} \in\{-1,1\}} \sum_{m_{\mu} \in J_{1}} \sum_{\substack{m_{k} \in J_{2} \\ k \neq \mu, \nu(\mu)}} \sum_{j \geq 0} \sum_{\substack{\gamma \in \ddot{G}_{i} \cap B(\mathbf{m}, \mu, \kappa, j) \\\left|\gamma_{i}\right| \leq\left|\gamma_{\mu}\right|, i=1, \ldots, s}} \frac{(\operatorname{det} \Gamma)^{-2} c_{2}^{2}}{(2 \pi)^{2 s}|\operatorname{Nm}(\gamma)|^{2}}
$$

Using (2.4.19), we obtain

$$
S_{1, \mu}\left(\ddot{G}_{i}\right)=O\left(\# J_{1} \# J_{2}^{s-2}\right)=O\left(i^{s-2} n^{((s-2) 4+2) / 9}\right) .
$$

Similarly we get that $S_{1, \mu}\left(\dot{G}_{0}\right)=O\left(n^{4(s-1) / 9}\right)$. Now from (2.4.15) and (2.3.8), we have

$$
\begin{aligned}
S_{1, \mu}(\tilde{G}) & =S_{1, \mu}\left(\dot{G}_{0}\right)+\sum_{1 \leq i \leq n^{5 / 9}+1} S_{1, \mu}\left(\ddot{G}_{i}\right) \\
& =O\left(\sum_{1 \leq i \leq n^{5 / 9}} i^{s-2} n^{((s-2) 4+2) / 9}\right)=O\left(n^{s-1-2 / 9}\right) .
\end{aligned}
$$

Hence, Lemma 8 is proved.

LEMma 9. With the notations as above

$$
\mathbf{E}\left[\left|\mathcal{A}\left(G^{(5)}\right)\right|^{2}\right]=O\left(n^{s-2}(\ln n)^{4}\right) .
$$

P r o of. Let

$$
G^{(5, \mu)}=\left\{\gamma \in G^{(5)}:\left|N_{\mu} \gamma_{\mu}\right| \leq 2^{(\ln n)^{4}} \text { and }\left|N_{j} \gamma_{j}\right|>2^{(\ln n)^{4}} \text { for } j<\mu\right\} \text {. }
$$

By (2.3.6), we have that

$$
G^{(5)}=\bigcup_{\mu \in[1, s]} G^{(5, \mu)}, \quad G^{(5, \mu)} \cap G^{(5, j)}=\emptyset \text { for } \mu \neq j .
$$

Similarly to (2.4.14)-(2.4.16), using the Cauchy-Schwartz inequality, we obtain from (2.1.2) and (2.4.1) that

$$
\mathbf{E}\left[\left|\mathcal{A}\left(G^{(5)}\right)\right|^{2}\right] \leq s 2^{s-1} \sum_{\mu \in[1, s]} \sum_{\substack{\kappa_{j} \in\{-1,1\} \\ j \in[1, s], j \neq \mu}} \sum_{\gamma^{(1)}, \boldsymbol{\gamma}^{(2)} \in G^{(5, \mu)}} \dot{\psi}\left(\boldsymbol{\gamma}^{(1)}, \gamma^{(2)}\right),
$$


with

$$
\dot{\psi}\left(\gamma^{(1)}, \gamma^{(2)}\right)=\frac{(\operatorname{det} \Gamma)^{-2}\left|\hat{\omega}\left(\tau \gamma^{(1)}\right)\right|\left|\hat{\omega}\left(\tau \gamma^{(2)}\right)\right|}{(2 \pi)^{2 s}\left|\operatorname{Nm}\left(\gamma^{(1)}\right)\right|\left|\operatorname{Nm}\left(\gamma^{(2)}\right)\right|} \tilde{\psi}\left(\gamma^{(1)}, \gamma^{(2)}\right)
$$

and

$$
\begin{aligned}
\tilde{\psi}\left(\gamma^{(1)}, \gamma^{(2)}\right)=\mid \mathbf{E}[ & \sin \left(\pi \theta_{\mu} N_{\mu} \gamma_{\mu}^{(1)}\right) \times \sin \left(\pi \theta_{\mu} N_{\mu} \gamma_{\mu}^{(2)}\right) \\
& \left.\times e\left(\sum_{1 \leq j \leq s, j \neq \mu}\left(\gamma_{j}^{(1)}-\gamma_{j}^{(2)}\right) \theta_{j} N_{j} \kappa_{j} / 2\right)\right] \mid .
\end{aligned}
$$

Hence

$$
\mathbf{E}\left[\left|\mathcal{A}\left(G^{(5)}\right)\right|^{2}\right] \leq s 2^{s} \sum_{\mu \in[1, s]} \sum_{\kappa_{j} \in\{-1,1\}, j \in[1, s], j \neq \mu}\left(\dot{S}_{1}(\mu)+\dot{S}_{2}(\mu)\right)
$$

with

$$
\dot{S}_{1}(\mu)=\sum_{\boldsymbol{\gamma} \in G^{(5, \mu)}} \dot{\psi}(\boldsymbol{\gamma}, \boldsymbol{\gamma}) \text { and } \quad \dot{S}_{2}(\mu)=\sum_{\substack{\gamma^{(1)}, \boldsymbol{\gamma}^{(2)} \in G^{(5, \mu)} \\ \boldsymbol{\gamma}^{(1)} \neq \boldsymbol{\gamma}^{(2)}}} \dot{\psi}\left(\boldsymbol{\gamma}^{(1)}, \gamma^{(2)}\right) .
$$

Bearing in mind that $|\sin (x)| \leq|x|$, we derive from (2.4.27) that

$$
\tilde{\psi}(\gamma, \gamma) \leq \min \left(1,\left|2 \pi N_{\mu} \gamma_{\mu}\right|^{2}\right)
$$

Consider $\dot{S}_{2}(\mu)$. By (2.4.11), we get for $\gamma^{(1)} \neq \gamma^{(2)}$ that

$$
\begin{aligned}
\tilde{\psi}\left(\gamma^{(1)}, \gamma^{(2)}\right) & \leq \prod_{j \in[1, s], j \neq \mu} \frac{1}{\pi N_{j}\left|\gamma_{j}^{(1)}-\gamma_{j}^{(2)}\right|} \\
& =\frac{N_{\mu}\left|\gamma_{\mu}^{(1)}-\gamma_{\mu}^{(2)}\right|}{\pi^{s-1} N\left|\operatorname{Nm}\left(\gamma^{(1)}-\gamma^{(2)}\right)\right|} \leq \frac{2^{(\ln n)^{4}+1}}{\pi^{s-1} C_{M}^{s} N} .
\end{aligned}
$$

According to (2.2.8), (2.3.6) and (1.4), we have

$$
G^{(5)} \subset \mathbb{G}\left(s \log _{2} C_{M}-(s-1)(n+1), n+1\right) .
$$

Using Lemma 3, we obtain $\# G^{(5)}=O\left(n^{s}\right)$. Applying (2.4.26) and (2.4.29), we get

$$
\dot{S}_{2}(\mu)=O\left(n^{2 s} N^{-1} 2^{(\ln n)^{4}+1}\right)=O(1) .
$$

Now we fix $\mu \in[1, s]$, and we consider $\dot{S}_{1}(\mu)$. Let

$$
\gamma \in \Gamma^{\perp} \cap B\left(\mathbf{m}, \mu, \boldsymbol{\kappa}^{(1)}, j\right) .
$$

According to (2.2.13) and (2.4.25), we have that

$$
\log _{2}\left|N_{\mu} \gamma_{\mu}\right|=\log _{2} N_{\mu}+m_{\mu}+z_{1} \leq(\ln n)^{4}, \quad z_{2} \in[0,1) .
$$


Hence

$$
m_{\mu} \in \dot{J}, \quad \text { with } \quad \dot{J}=\left(-\infty,(\ln n)^{4}-\log _{2} N_{\mu}\right] .
$$

By (2.4.17) and (1.4), we obtain that $C_{M}^{s} \max (1, j) \leq \mathrm{Nm}(\gamma) \leq(j+1) C_{M}^{s} 2^{s-1}$ and

$$
\begin{aligned}
& \sum_{\substack{m_{\mu} \in j \\
j \geq 0}} \sum_{\gamma \in G^{(5, \mu)} \cap B\left(\mathbf{m}, \mu, \boldsymbol{\kappa}^{(1)}, j\right)} \frac{\min \left(1,\left|N_{\mu} \gamma_{\mu}\right|^{2}\right)}{|\operatorname{Nm}(\gamma)|^{2}}=O\left(\sum_{m_{\mu} \in j} \sum_{j \geq 1} \frac{\min \left(1, N_{\mu}^{2} 2^{2 m_{\mu}}\right)}{j^{2}}\right) \\
& =O\left(\sum_{m_{\mu} \leq-\log _{j \geq 1} N_{\mu}} \frac{N_{\mu}^{2} 2^{2 m_{\mu}}}{j^{2}}+\sum_{m_{\mu} \in\left[-\log _{2}\right.} \sum_{\left.N_{\mu},(\ln n)^{4}-\log _{2} N_{\mu}\right]} \sum_{j \geq 1} \frac{1}{j^{2}}\right)=O\left((\ln n)^{4}\right) .
\end{aligned}
$$

Bearing in mind (2.2.13) and that $|\gamma| \leq N$, we have for $k \neq \mu, \nu(\mu)$ that

$$
m_{k}+z_{1}=\log _{2}\left|\gamma_{k}\right|=\log _{2} \operatorname{Nm}(\gamma)-\sum_{j \in[1, s], j \neq k} \log _{2}\left|\gamma_{j}\right| \geq \log _{2} C_{M}^{s}-(s-1)(n+1) \text {, }
$$

with $z_{1} \in[0,1)$ and

$$
m_{k} \in \ddot{J} \quad \text { with } \quad \ddot{J}=\left[\log _{2} C_{M}^{s}-(s-1)(n+1)-1, n+1\right] .
$$

By (2.2.15), (2.4.26), (2.4.29), 2.4.30) and (2.1.5), we get

$$
\begin{aligned}
& \dot{S}_{1}(\mu) \leq \bigcup_{\boldsymbol{\kappa}^{(1)} \in\{-1,1\}^{s}} \bigcup_{\substack{\mathbf{m} \in \mathbb{Z}^{s}, m_{1}+\cdots+m_{s}=0}} \bigcup_{j \geq 0} \sum_{\boldsymbol{\gamma \in G ^ { ( 5 , \mu ) } \cap B ( \mathbf { m } , \mu , \boldsymbol { \kappa } ^ { ( 1 ) } , j )}} \dot{\psi}(\boldsymbol{\gamma}, \boldsymbol{\gamma}) \\
& \leq \sum_{\boldsymbol{\kappa}^{(1)} \in\{-1,1\}^{s}} \sum_{m_{\mu} \in j} \sum_{\substack{m_{k} \in J, k \in[1, s], k \neq \mu, \nu(\mu)}} \sum_{j \geq 0} \sum_{\gamma \in G^{(5, \mu)} \cap B\left(\mathbf{m}, \mu, \boldsymbol{\kappa}^{(1)}, j\right)} \\
& \times \frac{(\operatorname{det} \Gamma)^{-2} c_{2}^{2} \min \left(1,\left|2 \pi N_{\mu} \gamma_{\mu}\right|^{2}\right)}{(2 \pi)^{2 s}|\operatorname{Nm}(\gamma)|^{2}} .
\end{aligned}
$$

Applying (2.4.32) and (2.4.33), we derive

$$
\dot{S}_{1}(\mu)=O\left(\# \ddot{J}^{s-2}(\ln n)^{4}\right)=O\left(n^{s-2}(\ln n)^{4}\right) .
$$

By (2.4.28) and (2.4.31), Lemma 9 is proved.

LEMMA 10. With the notations as above

$$
\mathcal{A}\left(G^{(4)}\right)=O(1)
$$


P r o o f. By (2.1.5), (2.1.2) and (2.4.1), we have

$$
\left|\mathcal{A}\left(G^{(4)}\right)\right| \leq(\operatorname{det} \Gamma)^{-1} c_{2} \sum_{\gamma \in G^{(4)}} \prod_{i=1}^{s} \frac{\mid \sin \left(\pi\left(\theta_{i} N_{i} \gamma_{i}\right) \mid\right.}{2 \pi\left|\gamma_{i}\right|}
$$

From (2.3.5), we get for $\gamma \in G^{(4)}$ that $|\gamma|>N$,

$$
\exists \nu \in[1, s] \quad \text { with } \quad \log _{2}\left(\left|\gamma_{\nu}\right|\right) \geq \log _{2}(|\gamma| / s) \geq n-1-\log _{2} s,
$$

and

$$
\log _{2}\left(\left|\gamma_{1}\right|\right)+\cdots+\log _{2}\left(\left|\gamma_{s}\right|\right) \leq 1 / 2 \log _{2} n, \quad n=\left[\log _{2} N\right]+1
$$

Hence, there exists $\mu \in\{1, \ldots, s\} \backslash\{\nu\}$ with

$$
\begin{aligned}
\log _{2}\left(\left|\gamma_{\mu}\right|\right) & \leq \frac{1}{s-1} \sum_{i \in[1, s], i \neq \nu} \log _{2}\left(\left|\gamma_{i}\right|\right) \\
& =\frac{1}{s-1}\left(\sum_{i \in[1, s]} \log _{2}\left(\left|\gamma_{i}\right|\right)-\log _{2}\left(\left|\gamma_{\nu}\right|\right)\right), \\
& \leq\left(-n+1+\log _{2} s+1 / 2 \log _{2} n\right) /(s-1)
\end{aligned}
$$

and

$$
\left|\gamma_{\mu}\right| \leq 4 N^{-\frac{1}{s-1}} n^{1 / 2}
$$

Bearing in mind that $N_{\mu} N^{-1 / s} \in\left[1 / c_{0}, c_{0}\right]$, we obtain

$$
\left|\sin \left(\pi\left(\theta_{\mu} N_{\mu} \gamma_{\mu}\right)\right)\right| \leq \pi\left|\theta_{\mu} N_{\mu} \gamma_{\mu}\right| \leq \pi\left|N_{\mu} \gamma_{\mu}\right|=O\left(N^{\frac{1}{s}-\frac{1}{s-1}} n^{1 / 2}\right)=O\left(n^{-s}\right) .
$$

Therefore

$$
\left|\mathcal{A}\left(G^{(4)}\right)\right|=O\left(n^{-s} \sum_{\gamma \in G^{(4)}} 1 /|\mathrm{Nm}(\gamma)|\right)=O\left(n^{-s} \# G^{(4)}\right) .
$$

Taking into account that $G^{(4)} \in \mathbb{G}(0,10 n)$, we get from Lemma 3 the assertion of Lemma 10

LEMma 11. There exists a real $w_{2}>0$ such that

$$
\begin{aligned}
\mathbf{E}\left[\left(\mathcal{R}\left(\boldsymbol{\theta} \cdot \mathbf{N} \cdot \mathbb{K}^{s}+\mathbf{x}, \Gamma\right)\right)^{2}\right] & \leq w_{2} n^{s-1}, \\
\mathbf{E}\left[\left|\mathcal{R}\left(\boldsymbol{\theta} \cdot \mathbf{N} \cdot \mathbb{K}^{s}+\mathbf{x}, \Gamma\right)-\mathcal{A}\left(G^{(1)}\right)\right|^{2}\right] & =O\left(n^{s-1-1 / 2}\right),
\end{aligned}
$$

and

$$
\mathbf{E}\left[\left(\mathcal{R}\left(\boldsymbol{\theta} \cdot \mathbf{N} \cdot \mathbb{K}^{s}+\mathbf{x}, \Gamma\right)-\sum_{i \in\left[1, n^{5 / 9}\right]} \mathcal{A}\left(\dot{G}_{i}\right)\right)^{2}\right]=O\left(n^{s-1-2 / 9}\right)
$$


Proof. By (2.4.6) and Lemma 1, we get

$$
\left|\mathcal{R}\left(\boldsymbol{\theta} \cdot \mathbf{N} \cdot \mathbb{K}^{s}+\mathbf{x}, \Gamma\right)-\sum_{i=1}^{5} \mathcal{A}\left(G^{(i)}\right)\right| \leq 2^{s} .
$$

It is easy to see that

$$
\left|\mathcal{R}\left(\boldsymbol{\theta} \cdot \mathbf{N} \cdot \mathbb{K}^{s}+\mathbf{x}, \Gamma\right)-\mathcal{A}\left(G^{(1)}\right)\right| \leq \sum_{i=2}^{5}\left|\mathcal{A}\left(G^{(i)}\right)\right|+2^{s} .
$$

Using the Cauchy-Schwartz inequality, we obatain

$$
\mathbf{E}\left[\left|\mathcal{R}\left(\boldsymbol{\theta} \cdot \mathbf{N} \cdot \mathbb{K}^{s}+\mathbf{x}, \Gamma\right)-\mathcal{A}\left(G^{(1)}\right)\right|^{2}\right] \leq 5\left(\sum_{i=2}^{5} \mathbf{E}\left[\left|\mathcal{A}\left(G^{(i)}\right)\right|^{2}\right]+2^{2 s}\right) .
$$

Applying Lemma 5-Lemma 10, we have (2.4.36). By Lemma 7, the triangle inequality and the Cauchy-Schwartz inequality, we get (2.4.35).

Now consider the statement (2.4.37). From (2.3.9) and (2.4.1) we obtain, that

$$
\mathcal{A}\left(G^{(1)}\right)=\sum_{i=1}^{\left[n^{5 / 9}\right]} \mathcal{A}\left(\dot{G}_{i}\right)+\mathcal{A}(\tilde{G}), \quad \text { with } \quad \tilde{G}=\dot{G}_{0} \bigcup_{i=1}^{\left[n^{5 / 9}\right]} \ddot{G}_{i}
$$

According to (2.4.14), we have

$$
\mathbf{E}\left[|\mathcal{A}(\tilde{G})|^{2}\right] \leq 2^{2 s} \sum_{\mu \in[1, s]} S_{1, \mu}(\tilde{G})+\frac{2^{2 s}}{\pi^{s} C_{M}^{s} N} S_{2}(\tilde{G}, \tilde{G}) .
$$

Using Lemma 8 and (2.4.24), we derive

$$
\mathbf{E}\left[|\mathcal{A}(\tilde{G})|^{2}\right]=O\left(n^{s-1-2 / 9}\right) .
$$

From (2.4.36) and the triangle inequality, we get (2.4.37). Therefore, Lemma 11] is proved.

\subsection{Lower bound of variance of $\mathcal{R}\left(\boldsymbol{\theta} \cdot \mathbf{N} \cdot \mathbb{K}^{s}+\mathbf{x}, \Gamma\right)$}

The main idea of the proof of Lemma 12 is to choose in $G_{1} c_{0} n^{s-1}$ different blocks $\mathcal{D}(\mathbf{m})$ of volume $2 \operatorname{det}(\Gamma)$ and lying under the hyperbole $\left\{\mathbf{x} \in \mathbb{R}^{s}|| x_{1} \cdots x_{s} \mid<\operatorname{det}(\Gamma)\right\}$. The next step is to prove that for given $\gamma \in \mathcal{D}(\mathbf{m})$ the corresponding summands in (2.5.1) are sufficiently large. This follows from the statement $\min _{i}\left|N_{i} \gamma_{i}\right| \geq 1$ and the obvious inequality

$$
\max (|\cos (y)|,|\cos 2 y|) \geq 1 / 2 \text { for all } y \text {. }
$$

LEMma 12. There exist reals $w_{3}, c_{5}>0$ such that for $N>c_{5}$ and for all $\mathbf{x}$ 
GAUSSIAN LIMITING DISTRIBUTION OF LATTICE POINTS IN PARALLEPIDED

$$
\begin{aligned}
\check{\psi}:=\sum_{\gamma \in G^{(1)}} \frac{(\operatorname{det} \Gamma)^{-2}}{(2 \pi)^{2 s}|\operatorname{Nm}(\boldsymbol{\gamma})|^{2}} \mathbf{E}\left[\prod_{i=1}^{s} \sin ^{2}(\right. & \left.\left.\pi \theta_{i} N_{i} \gamma_{i}\right)\right] \\
& \times 2 \cos ^{2}(2 \pi\langle\boldsymbol{\gamma}, \mathbf{x}\rangle) \geq w_{3} n^{s-1}
\end{aligned}
$$

Proof. Let $\mathbf{m} \in \mathbb{Z}^{s}, m_{1}+\cdots+m_{s}=0, q=2+\left[\operatorname{det} \Gamma^{\perp} / C_{M}^{s}\right]$, and

$$
\mathcal{D}(\mathbf{m})=\prod_{i=1}^{s}\left[-q^{m_{i}}, q^{m_{i}}\right] \times\left[-\operatorname{det} \Gamma^{\perp} q^{m_{s}}, \operatorname{det} \Gamma^{\perp} q^{m_{s}}\right] .
$$

According to Minkowsky's theorem, there exists

$$
\gamma(\mathbf{m}) \in \Gamma^{\perp} \backslash\{0\} \quad \text { with } \quad \gamma(\mathbf{m}) \in \mathcal{D}(\mathbf{m}) .
$$

We see that

$$
|\operatorname{Nm}(\gamma(\mathbf{m}))| \leq \operatorname{det} \Gamma^{\perp} .
$$

Suppose $\left|\gamma(\mathbf{m})_{i}\right| \leq q^{m_{i}-1}$ for some $i \in[1, s-1]$. By (1.4) we get

$$
C_{M}^{s} \leq|\operatorname{Nm}(\gamma(\mathbf{m}))| \leq \operatorname{det} \Gamma^{\perp} / q<C_{M}^{s} .
$$

We arrive at a contradiction. Hence

$$
\left|\gamma(\mathbf{m})_{i}\right| \in\left(q^{m_{i}-1}, q^{m_{i}}\right] \text { for } i \in[1, s-1], \quad \text { and } \quad \gamma\left(\mathbf{m}^{(1)}\right) \neq \gamma\left(\mathbf{m}^{(2)}\right),
$$

for $\mathbf{m}^{(1)} \neq \mathbf{m}^{(2)}$. Let

$$
\bar{G}=\left\{\gamma(\mathbf{m}) \mid-n /(4 s) \log _{q} 2 \leq m_{i} \leq-2 s, \quad i=1, \cdots, s-1\right\} .
$$

We see for sufficiently large $N$ that

$$
\# \bar{G} \geq n^{s-1}\left((5 s)^{-1} \log _{q} 2\right)^{s-1} .
$$

By (2.2.5) $N_{i} N^{1 / s} \in\left[1 / c_{0}, c_{0}\right]$. From (2.5.4), we obtain for sufficiently large $N$ that

$$
N_{i} 2^{-2 s} \geq\left|N_{i} \gamma_{i}\right| \geq c_{0}^{-1} 2^{n / s-n /(4 s) \log _{q} 2-2} \geq 2^{\ln ^{4} n}, \quad i \in[1, s-1], \quad \gamma \in \bar{G} .
$$

Consider $\gamma_{s}$ with $\gamma \in \bar{G}$. By (2.5.2), we have

$$
\left|\gamma_{s}\right|=\left|\operatorname{Nm}(\gamma)\left(\gamma_{1} \cdots \gamma_{s-1}\right)^{-1}\right| \in\left|\gamma_{1} \cdots \gamma_{s-1}\right|^{-1}\left[C_{M}^{s}, \operatorname{det} \Gamma^{\perp}\right] \text {. }
$$

Now using (2.5.3) and (2.5.4), we obtain for sufficiently large $N$ that

$$
\log _{q}\left|N_{s} \gamma_{s}\right| \leq n / s \log _{q} 2+\log _{q}\left(c_{0} \operatorname{det} \Gamma^{\perp}\right)-m_{1}-\cdots m_{s-1} \leq 3 / 4 n \log _{q} 2,
$$

and

$\log _{q}\left|N_{s} \gamma_{s}\right| \geq(n-1) / s \log _{q} 2-\log _{q} c_{0}+\log _{q} C_{M}^{s}-m_{1}-\cdots-m_{s-1}-s \geq \frac{n\left(\log _{q} 2\right)}{2 s}$.

Therefore, we get for sufficiently large $N$ and for $\gamma \in \bar{G}$

$$
|\gamma|<N / 2,|\mathrm{Nm}(\gamma)| \leq \operatorname{det} \Gamma^{\perp},\left|N_{i} \gamma_{i}\right| \geq 2^{\ln ^{4} n}, i=1, \ldots, s .
$$


So $\bar{G} \cup 2 \bar{G} \subset G_{1}($ see $(2.3 .4)$ ).

Let $\gamma \in \bar{G}$. Taking into account that $\left|N_{i} \gamma_{i}\right| \geq 4(i=1, \ldots, s)$, we obtain

$$
\int_{0}^{1} \sin ^{2}\left(\pi \theta_{i} N_{i} \gamma_{i}\right) \mathrm{d} \theta_{\mathrm{i}}=1 / 2-1 / 2 \int_{0}^{1} \cos \left(2 \pi \theta_{\mathrm{i}} \mathrm{N}_{\mathrm{i}} \gamma_{\mathrm{i}}\right) \mathrm{d} \theta_{\mathrm{i}} \geq 1 / 4
$$

Let $I=[1 / 6,1 / 3] \cup[2 / 3,5 / 6]$. If $\{\langle\boldsymbol{\gamma}, \mathbf{x}\rangle\} \notin I$, then $|\cos (2 \pi\{\langle\boldsymbol{\gamma}, \mathbf{x}\rangle\})| \geq 1 / 2$. Let $\{\langle\gamma, \mathbf{x}\rangle\} \in I$. Then we take $2 \gamma$ instead of $\gamma$. We see that

$$
|\cos (2 \pi\{\langle 2 \gamma, \mathbf{x}\rangle\})| \geq 1 / 2
$$

and

$$
\max \left(\cos ^{2}(2 \pi\langle\gamma(\mathbf{m}), \mathbf{x}\rangle), \cos ^{2}(2 \pi\langle 2 \gamma(\mathbf{m}), \mathbf{x}\rangle)\right) \geq 1 / 4
$$

By (2.5.1)-(2.5.8), we have

$$
\begin{gathered}
\check{\psi} \geq \sum_{\substack{\gamma(\mathbf{m}) \in \bar{G} \\
\mathbf{m} \in \mathbb{Z}^{s-1}}} \frac{(\operatorname{det} \Gamma)^{-2}}{(2 \pi)^{2 s} C_{M}^{2 s}}(1 / 4)^{s-1} \max \left(\cos ^{2}(2 \pi\langle\gamma(\mathbf{m}), \mathbf{x}\rangle), \cos ^{2}(2 \pi\langle 2 \gamma(\mathbf{m}), \mathbf{x}\rangle)\right) \\
\geq w_{4} \# \bar{G}, \quad \text { with } w_{4}=(\operatorname{det} \Gamma)^{-2}\left((2 \pi)^{2 s} C_{M}^{2 s}\right)^{-1} 4^{-s} .
\end{gathered}
$$

Applying (2.5.5), we get the assertion of Lemma 12.

LEMMA 13. There exist reals $c_{6}, w_{1}>0$ such that for $N>c_{6}$

$$
\mathbf{E}\left[\left(\mathcal{R}\left(\boldsymbol{\theta} \cdot \mathbf{N} \cdot \mathbb{K}^{s}+\mathbf{x}, \Gamma\right)\right)^{2}\right] \geq w_{1} n^{s-1} .
$$

P r o o f. Applying (2.1.2) and (2.4.1), we have

$$
\mathbf{E}\left[\left|\mathcal{A}\left(G^{(1)}, 0\right)\right|^{2}\right]=\ddot{S}_{1}+\ddot{S}_{2},
$$

with

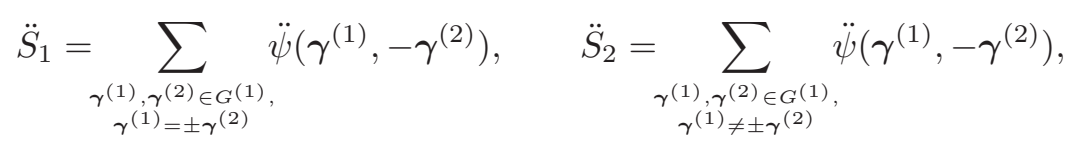

where

$$
\ddot{\psi}\left(\gamma^{(1)},-\gamma^{(2)}\right)=\frac{(\operatorname{det} \Gamma)^{-2} \hat{\omega}\left(\tau \gamma^{(1)}\right) \hat{\omega}\left(-\tau \gamma^{(2)}\right)}{(2 \pi)^{2 s} \operatorname{Nm}\left(\gamma^{(1)}\right) \operatorname{Nm}\left(-\gamma^{(2)}\right)} e\left(\left\langle\gamma^{(1)}-\gamma^{(2)}\right\rangle, x\right) \breve{\psi}\left(\gamma^{(1)}-\gamma^{(2)}\right) .
$$

and

$$
\breve{\psi}\left(\gamma^{(1)}, \gamma^{(2)}\right)=\mathbf{E}\left[\prod_{i=1}^{s} \sin \left(\pi \theta_{i} N_{i} \gamma_{i}^{(1)}\right) \sin \left(-\pi \theta_{i} N_{i} \gamma_{i}^{(2)}\right)\right] .
$$

We consider $\ddot{S}_{1}$. Bearing in mind (2.1.3), (2.1.4), that

$$
|e(z)-1|=2|\sin (\pi z)| \leq 2 \pi|z|
$$


and that $\omega(\mathbf{x})$ is supported inside the unit ball $B=\{\mathbf{x}:|\mathbf{x}| \leq 1\}$, we obtain for $\tau=1 / N^{2}$ and $|\gamma| \leq N$ that

$$
\begin{aligned}
|\hat{\omega}(\tau \gamma)-1| & =\left|\int_{\mathbb{R}^{s}} e(\langle\tau \gamma, \mathbf{x}\rangle) \omega(\mathbf{x}) \mathrm{d} \mathbf{x}-1\right|=\left|\int_{\mathrm{B}}\left(\mathrm{e}\left(\langle\boldsymbol{\gamma}, \mathbf{x}\rangle / \mathrm{N}^{2}\right)-1\right) \omega(\mathbf{x}) \mathrm{d} \mathbf{x}\right| \\
& \leq\left|\int_{B}\right| e\left(\langle\gamma, \mathbf{x}\rangle / N^{2}\right)-1|\omega(\mathbf{x}) \mathrm{d} \mathbf{x}| \\
& \leq 2 \pi / N \int_{B} \omega(\mathbf{x}) \mathrm{d} \mathbf{x}=2 \pi / \mathrm{N}
\end{aligned}
$$

By (2.5.12), we see that

$$
\breve{\psi}\left(\gamma^{(1)},-\gamma^{(2)}\right)\left(\operatorname{Nm}\left(\gamma^{(1)}\right) \operatorname{Nm}\left(-\gamma^{(2)}\right)\right)^{-1}=\breve{\psi}\left(\gamma^{(1)}, \gamma^{(2)}\right)\left(\operatorname{Nm}\left(\gamma^{(1)}\right) \operatorname{Nm}\left(\gamma^{(2)}\right)\right)^{-1}
$$

Taking into account that $1+\cos (2 z)=2 \cos ^{2}(z)$, we get from (2.5.1), (2.5.11), (2.5.13) and (2.5.14) that

$$
\ddot{S}_{1}=\check{\psi}+O\left(1 / N \sum_{\gamma \in G^{(1)}} \frac{1}{|\mathrm{Nm}(\gamma)|^{2}}\right) .
$$

By (2.4.15), 2.4.23), 2.5.13) and Lemma 12, we have for sufficiently large $N$

$$
\ddot{S}_{1} \geq 0.5 w_{3} n^{s-1} \text {. }
$$

Now we consider $\ddot{S}_{2}$. We see from (2.5.12) and (2.4.11)

$$
\begin{aligned}
& \left|\breve{\boldsymbol{\psi}}\left(\gamma^{(1)}, \gamma^{(2)}\right)\right| \\
& =\left|2^{-2 s} \sum_{\kappa_{1}^{(1)}, \ldots, \kappa_{s}^{(2)} \in\{-1,1\}} \kappa_{1}^{(1)} \kappa_{2}^{(1)} \cdots \kappa_{s}^{(2)} \mathbf{E}\left[e\left(\sum_{1 \leq i \leq s, j=1,2} \theta_{i} \times N_{i} \kappa_{i}^{(j)} \gamma_{i}^{(j)} / 2\right)\right]\right| \\
& \leq 2^{-s} \sum_{\kappa_{1}, \ldots, \kappa_{s} \in\{-1,1\}}\left|\mathbf{E}\left[e\left(\sum_{i=1}^{s} \theta_{i} N_{i}\left(\gamma_{i}^{(1)}+\kappa_{i} \gamma_{i}^{(2)}\right) / 2\right)\right]\right| \\
& \leq 2^{-s} \sum_{\kappa_{1}, \ldots, \kappa_{s} \in\{-1,1\}} \prod_{i=1}^{s} \min \left(1, \frac{1}{\pi\left|N_{i}\left(\gamma_{i}^{(1)}+\kappa_{i} \gamma_{i}^{(2)}\right)\right|}\right) .
\end{aligned}
$$

Applying Lemma 4, we get that $\psi\left(\gamma^{(1)}, \gamma^{(2)}\right)=O\left(n^{-20 s}\right)$. By (2.4.16), (2.4.24) and (2.5.11), we derive that

$$
\ddot{S}_{2}=O\left(n^{-20 s} S_{2}\left(G^{(1)}, G^{(1)}\right)\right)=O\left(n^{-2 s}\right) .
$$


From (2.5.10) and (2.5.16), we have for sufficiently large $N$ that

$$
\mathbf{E}\left[\left|\mathcal{A}\left(G^{(1)}, 0\right)\right|^{2}\right] \geq 0.25 w_{3} n^{s-1} .
$$

By the triangle inequality, we obtain

$$
\begin{aligned}
& \left(\mathbf{E}\left[\left(\mathcal{R}\left(\boldsymbol{\theta} \cdot \mathbf{N} \cdot \mathbb{K}^{s}+\mathbf{x}, \Gamma\right)\right)^{2}\right]\right)^{1 / 2} \\
& \geq\left(\mathbf{E}\left[\left|\mathcal{A}\left(G^{(1)}, 0\right)\right|^{2}\right]\right)^{1 / 2}-\left(\mathbf{E}\left[\left|\mathcal{R}\left(\boldsymbol{\theta} \cdot \mathbf{N} \cdot \mathbb{K}^{s}+\mathbf{x}, \Gamma\right)-\mathcal{A}\left(G^{(1)}, 0\right)\right|^{2}\right]\right)^{1 / 2}
\end{aligned}
$$

Using (2.5.18) and Lemma 11, we get the assertion of Lemma 13 .

\subsection{Four moments estimates for $\mathcal{A}\left(\dot{G}_{i}\right)$}

Let

$$
\delta(\mathfrak{T})= \begin{cases}1, & \text { if } \mathfrak{T} \text { is true } \\ 0, & \text { otherwise }\end{cases}
$$

LEMMA 14. With the notations as above, we have

$$
\mathbf{E}\left[\left|\mathcal{A}\left(\dot{G}_{i}\right)\right|^{4}\right]=O\left(i^{2(s-2)} n^{8 / 9(s-1)}\right) .
$$

P r o of. Using the following simple inequality

$$
\left|\sum_{1 \leq i \leq 2^{s}} a_{i}\right|^{4} \leq\left(2^{s} \max _{1 \leq i \leq 2^{s}}\left|a_{i}\right|\right)^{4} \leq 2^{4 s} \sum_{1 \leq i \leq 2^{s}}\left|a_{i}\right|^{4}
$$

we obtain from (2.4.4)

$$
\mathbf{E}\left[\left|\mathcal{A}\left(\dot{G}_{i}, \tau\right)\right|^{4}\right] \leq 2^{4 s} \sum_{\kappa_{1}, \ldots, \kappa_{s} \in\{-1,1\}}\left|\mathcal{B}\left(\dot{G}_{i}, \kappa, \tau\right)\right|^{4} .
$$

Applying (2.4.8) and Lemma 6. we get

$$
\mathbf{E}\left[\mid \mathcal{B}\left(\left.\left(\dot{G}_{i}, \boldsymbol{\kappa}\right)\right|^{4}\right)\right]=\prod_{1 \leq j \leq 4} \sum_{\boldsymbol{\gamma}^{(j)} \in \dot{G}_{i}}\left|h\left(\boldsymbol{\gamma}^{(j)}\right)\right|\left(\delta(\hat{\gamma}=\mathbf{0})+(1-\delta(\hat{\gamma}=\mathbf{0})) N^{-1} O(1)\right)
$$

where $\hat{\gamma}=\gamma^{(1)}-\gamma^{(2)}+\gamma^{(3)}-\gamma^{(4)}$. From (2.4.7), 2.4.16), 2.4.24) and Lemma 3 , we derive that

$$
\mathbf{E}\left[\mid \mathcal{B}\left(\left.\left(\dot{G}_{i}, \boldsymbol{\kappa}, \tau\right)\right|^{4}\right)\right]=V_{1}+V_{2}+O\left(n^{8 s} / N\right),
$$

where

$$
V_{k}=\prod_{1 \leq j \leq 4} \sum_{\boldsymbol{\gamma}^{(j)} \in \dot{G}_{i}} \frac{(\operatorname{det} \Gamma)^{-1}\left|\hat{\omega}\left(\tau \boldsymbol{\gamma}^{(j)}\right)\right|}{(2 \pi)^{s}\left|\operatorname{Nm}\left(\boldsymbol{\gamma}^{(j)}\right)\right|} \delta(\hat{\gamma}=\mathbf{0}) \delta_{k}(\bar{\gamma}), \quad k=1,2,
$$


with $\bar{\gamma}=\left(\gamma^{(1)}, \gamma^{(2)}, \gamma^{(3)}, \gamma^{(4)}\right)$

$\delta_{1}(\bar{\gamma})=\delta\left(\nexists j, l \in[1,4], j \neq l \mid \gamma^{(j)}=(-1)^{l-j+1} \gamma^{(l)}\right), \quad$ and $\quad \delta_{2}(\bar{\gamma})=1-\delta_{1}(\bar{\gamma})$.

By (2.2.6) and (2.2.10), we have that

$$
\gamma^{(j)}=\tilde{\gamma}^{(j)} \cdot \sigma\left(\eta^{(j)}\right) \quad \text { with } \quad \tilde{\gamma}^{(j)}=\left(\tilde{\gamma}_{1}^{(j)}, \ldots, \tilde{\gamma}_{s}^{(j)}\right), \tilde{\gamma}^{(j)} \in \mathbb{F}_{n}
$$

and

$$
\eta^{(j)} \in \dot{\mathfrak{U}}\left(\gamma^{(j)}, a, b\right),(j=1, \ldots, 4)
$$

Using (2.2.10) and (2.3.8), we obtain that

Hence

$$
a=i n^{4 / 9} \text { and } \quad b=n^{4 / 9}-n^{2 / 9} .
$$

$$
\begin{aligned}
V_{k}= & O\left(\prod_{1 \leq j \leq 4} \sum_{\tilde{\boldsymbol{\gamma}}^{(j)} \in \mathbb{F}_{n}} \sum_{\eta^{(j)} \in \dot{\mathfrak{U}}\left(\tilde{\boldsymbol{\gamma}}^{(j)}, a, b\right)} \frac{1}{\left|\operatorname{Nm}\left(\tilde{\boldsymbol{\gamma}}^{(j)}\right)\right|} \delta_{k}(\bar{\gamma})\right) \\
& \times \delta\left(\tilde{\boldsymbol{\gamma}}^{(1)} \cdot \sigma\left(\eta^{(1)}\right)-\tilde{\boldsymbol{\gamma}}^{(2)} \cdot \sigma\left(\eta^{(2)}\right)+\tilde{\boldsymbol{\gamma}}^{(3)} \cdot \sigma\left(\eta^{(3)}\right)-\tilde{\boldsymbol{\gamma}}^{(4)} \cdot \sigma\left(\eta^{(4)}\right)=\mathbf{0}\right) .
\end{aligned}
$$

It is easy to see that

$$
\tilde{\gamma}^{(1)} \cdot \sigma\left(\eta^{(1)}\right)-\tilde{\gamma}^{(2)} \cdot \sigma\left(\eta^{(2)}\right)+\tilde{\gamma}^{(3)} \cdot \sigma\left(\eta^{(3)}\right)-\tilde{\gamma}^{(4)} \cdot \sigma\left(\eta^{(4)}\right)=\mathbf{0}
$$

if and only if

$$
\left(\tilde{\gamma}_{1}^{(1)} \sigma_{1}\left(\eta^{(1)}\right)-\tilde{\gamma}_{1}^{(2)} \sigma_{1}\left(\eta^{(2)}\right)+\tilde{\gamma}_{1}^{(3)} \sigma_{1}\left(\eta^{(3)}\right)\right) / \tilde{\gamma}_{1}^{(4)} \sigma_{1}\left(\eta^{(4)}\right)=1 .
$$

First we consider $V_{1}$. We fix $\tilde{\gamma}^{(1)}, \tilde{\gamma}^{(2)}, \tilde{\gamma}^{(3)}, \tilde{\gamma}^{(4)}$ and $\eta^{(4)}$. From (2.2.6) and (2.6.5), we get that there is no degenerate solutions $\left(\eta^{(1)}, \eta^{(2)}, \eta^{(3)}\right)$ of the equation (2.6.7). Applying Theorem A, we have that the number of non-degenerate solutions $\left(\eta^{(1)}, \eta^{(2)}, \eta^{(3)}\right)$ of (2.6.7) is finite. Hence

$$
V_{1}=O\left(\sum_{\tilde{\gamma}^{(j)} \in \mathbb{F}_{n}, 1 \leq j \leq 4} \sum_{\eta^{(4)} \in \dot{\mathfrak{U}}\left(\tilde{\gamma}^{(4)}, a, b\right)} \frac{1}{\left|\operatorname{Nm}\left(\tilde{\gamma}^{(1)}\right)\right| \cdots\left|\operatorname{Nm}\left(\tilde{\gamma}^{(4)}\right)\right|}\right) .
$$

By (2.2.11) and (2.2.12), we derive

$$
V_{1}=O\left((\ln n)^{4} b(a+b)^{s-2}\right)=O\left(i^{s-2} n^{4 / 9(s-1)}(\ln n)^{4}\right) .
$$

Now we consider $V_{2}$. Let $\gamma^{\left(j_{0}\right)}=(-1)^{l_{0}-j_{0}+1} \gamma^{\left(l_{0}\right)}$. Bearing in mind that $\hat{\gamma}=\gamma^{(1)}-\gamma^{(2)}+\gamma^{(3)}-\gamma^{(4)}=\mathbf{0}$, we obtain that $\boldsymbol{\gamma}^{\left(j_{1}\right)}=(-1)^{l_{1}-j_{1}+1} \boldsymbol{\gamma}^{\left(l_{1}\right)}$ 
with $\left\{j_{1}, l_{1}\right\}=\{1,2,3,4\} \backslash\left\{j_{0}, l_{0}\right\}$. Hence, from (2.6.4), we get

$$
V_{2}=O\left(\left(\sum_{\tilde{\boldsymbol{\gamma}}^{(1)} \in \mathbb{F}_{n}} \sum_{\eta^{(1)} \in \dot{\mathfrak{U}}\left(\tilde{\boldsymbol{\gamma}}^{(1)}, a, b\right)} \frac{1}{\mathrm{Nm}^{2}\left(\tilde{\boldsymbol{\gamma}}^{(1)}\right)}\right)^{2}\right) .
$$

By (2.2.11) and (2.2.12), we have

$$
V_{2}=O\left(b^{2}(a+b)^{2(s-2)}\right)=O\left(i^{2(s-2)} n^{8 / 9(s-1)}\right) .
$$

Using (2.6.2), (2.6.3) and (2.6.9), we obtain (2.6.1) and the assertion of Lemma 14.

\subsection{Conditional variance estimate}

From (2.8.8) and (2.8.9), we get that the bound in the martingale CLT depends on $\mathbb{A}_{n}=E\left(\left|\mathbb{V}_{n}^{2}-1\right|\right)$, where $\mathbb{V}_{n}^{2}$ is a Lévy conditional variance. By (2.8.16) and (2.8.20), in order to obtain $\mathbb{A}_{n}$, it is sufficient to find the upper bound of $\varkappa$ (see (2.7.12) ). We will obtain this bound in Lemma 17 by using the auxiliary variables $H_{i, j}, \dot{H}_{i, j}$ and $\ddot{H}_{i, j}$, with $H_{i, j}=\dot{H}_{i, j}+\ddot{H}_{i, j}$. In Lemma 15 , we prove that $\ddot{H}_{i, j}$ is the essential part of $H_{i, j}$. In order to obtain the upper bound of $\ddot{H}_{i, j}$, we decompose the domain of the summation by using the auxiliary functions $\delta_{i}(l)$ (see (2.7.1)-(2.7.2)) Next we use Diophantine inequalities (2.3.2) and (2.3.3) (see Lemma 16).

Let

and

$$
\begin{aligned}
& \bar{\gamma}=\left(\gamma^{(1)}, \gamma^{(2)}, \gamma^{(3)}, \gamma^{(4)}\right), \\
& \dot{\gamma}=\dot{\gamma}=\left(\dot{\gamma}_{1}, \ldots, \dot{\gamma}_{s}\right)=\boldsymbol{\kappa}^{(1)} \cdot \gamma^{(1)}+\cdots+\boldsymbol{\kappa}^{(4)} \cdot \boldsymbol{\gamma}^{(4)}, \\
& \ddot{\gamma}=\boldsymbol{\kappa}^{(3)} \cdot \boldsymbol{\gamma}^{(1)}+\boldsymbol{\kappa}^{(4)} \cdot \boldsymbol{\gamma}^{(2)}, \\
& \ddot{\gamma}=\ddot{\gamma}=\left(\ddot{\gamma}_{1}, \ldots, \ddot{\gamma}_{s}\right)=\boldsymbol{\kappa}^{(3)} \cdot \boldsymbol{\gamma}^{(1)}+\boldsymbol{\kappa}^{(4)} \cdot \boldsymbol{\gamma}^{(2)},
\end{aligned}
$$

$$
\begin{aligned}
& \dot{\delta}_{1}(\bar{\gamma})=\delta(\dot{\gamma}=\mathbf{0}, \ddot{\gamma}=\mathbf{0}), \\
& \dot{\delta}_{2}(\bar{\gamma})=\delta(\dot{\gamma}=\mathbf{0}, \ddot{\gamma} \neq 0), \\
& \dot{\delta}_{3}(\bar{\gamma})=\delta(\dot{\gamma} \neq \mathbf{0}, \ddot{\gamma}=\mathbf{0}), \\
& \dot{\delta}_{4}(\bar{\gamma})=\delta\left(\dot{\gamma} \neq \mathbf{0}, \ddot{\gamma} \neq \mathbf{0}, \exists \nu \in[1, s] \dot{\gamma}_{\nu}=0 \text { and } \quad \ddot{\gamma}_{\nu}=0\right), \\
& \dot{\delta}_{5}(\bar{\gamma})=\delta\left(\dot{\gamma} \neq \mathbf{0}, \ddot{\gamma} \neq \mathbf{0}, \exists \nu \in[1, s] \dot{\gamma}_{\nu}=0 \text { and } \nexists \nu \in[1, s] \quad \dot{\gamma}_{\nu}=0, \ddot{\gamma}_{\nu}=0\right), \\
& \dot{\delta}_{6}(\bar{\gamma})=\delta\left(\dot{\gamma} \neq \mathbf{0}, \ddot{\gamma} \neq \mathbf{0}, \forall \nu \in[1, s] \dot{\gamma}_{\nu} \neq 0 \text { and } \quad \ddot{\gamma}_{\nu} \neq 0\right), \\
& \dot{\delta}_{7}(\bar{\gamma})=\delta\left(\dot{\gamma} \neq \mathbf{0}, \ddot{\gamma} \neq \mathbf{0}, \forall \nu \in[1, s] \dot{\gamma}_{\nu} \neq 0 \text { and } \exists \nu \in[1, s] \quad \ddot{\gamma}_{\nu}=0\right) .
\end{aligned}
$$


It is easy to verify that

$$
\sum_{1 \leq k \leq 7} \dot{\delta}_{k}(\bar{\gamma})=1
$$

Lemma 15. Let $l \geq 2, i, j=1,2, \ldots$, and let

$$
\begin{aligned}
\dot{H}_{i, j}(l)= & \sum_{\boldsymbol{\kappa}^{(1)}, \ldots, \boldsymbol{\kappa}^{(4)} \in\{-1,1\}^{s} \boldsymbol{\gamma}^{(1)}, \boldsymbol{\gamma}^{(2)} \in \dot{G}_{i} \boldsymbol{\gamma}^{(3)}, \boldsymbol{\gamma}^{(4)} \in \dot{G}_{j}} h\left(\boldsymbol{\gamma}^{(1)}\right) \cdots h\left(\boldsymbol{\gamma}^{(4)}\right) \\
& \times \dot{\delta}_{l}(\overline{\boldsymbol{\gamma}}) \mathbf{E}\left[e\left(\sum_{k \in[1, s]} \sum_{\nu \in[1,2]} \theta_{k} \kappa_{k}^{(\nu)} \gamma_{k}^{(\nu)} N_{k} / 2\right)\right] \\
& \times \mathbf{E}\left[e\left(\sum_{k \in[1, s]} \sum_{\nu \in[3,4]} \theta_{k} \kappa_{k}^{(\nu)} \gamma_{k}^{(\nu)} N_{k} / 2\right)\right] .
\end{aligned}
$$

Then

$$
\dot{H}_{i, j}(l)=O\left(n^{-10 s}\right) .
$$

P r o of. Applying (2.4.7) and Lemma 6, we get

$$
\begin{array}{r}
\dot{H}_{i, j}(l)=O\left(\sum_{\boldsymbol{\kappa}^{(1)}, \ldots, \boldsymbol{\kappa}^{(4)} \in\{-1,1\}^{s}} \sum_{\boldsymbol{\gamma}^{(1)}, \boldsymbol{\gamma}^{(2)} \in \dot{G}_{i}} \sum_{\boldsymbol{\gamma}^{(3)}, \boldsymbol{\gamma}^{(4)} \in \dot{G}_{j}}\left|\mathrm{Nm}\left(\boldsymbol{\gamma}^{(1)} \cdots \boldsymbol{\gamma}^{(4)}\right)\right|^{-1} \dot{\delta}_{l}(\bar{\gamma})\right. \\
\left.\times \prod_{k \in[1, s]} \min \left(1, \frac{1}{N_{k}\left|\dot{\gamma}_{k}-\ddot{\gamma}_{k}\right|}\right) \min \left(1, \frac{1}{N_{k}\left|\ddot{\gamma}_{i}\right|}\right)\right) .
\end{array}
$$

From (2.7.1), we have that for $l \geq 2$ there exists $k_{0} \in[1, s]$ such that

$$
\max \left(\left|\dot{\gamma}_{k_{0}}-\ddot{\gamma}_{k_{0}}\right|,\left|\ddot{\gamma}_{k_{0}}\right|\right)>0 \text {. }
$$

Using Lemma 4, we derive that $N_{i} \max \left(\left|\dot{\gamma}_{k_{0}}-\ddot{\gamma}_{k_{0}}\right|,\left|\ddot{\gamma}_{k_{0}}\right|\right)>\dot{c} n^{20 s}$. Thus

$$
\dot{H}_{i, j}(l)=O\left(n^{-20 s}\left(\sum_{\gamma \in G^{(1)}}(\mathrm{Nm}(\gamma))^{-1}\right)^{4}\right) \text {. }
$$

By (2.2.8) and (2.3.4) $G^{(1)}=\mathbb{G}(0,2 n)$. Similarly to (2.6.8) and (2.6.9), we obtain from Lemma 3 that

$$
\dot{H}_{i, j}(l)=O\left(n^{-20 s} n^{8 s}\right)=O\left(n^{-10 s}\right) .
$$

Hence, Lemma 15 is proved. 
LEMMA 16. Let $l \geq 2, i<j$, and

$$
\begin{array}{r}
\ddot{H}_{i, j}(l)=\sum_{\boldsymbol{\kappa}^{(1)}, \ldots, \boldsymbol{\kappa}^{(4)} \in\{-1,1\}^{s} \boldsymbol{\gamma}^{(1)}, \boldsymbol{\gamma}^{(2)} \in \dot{G}_{i}} \sum_{\boldsymbol{\gamma}^{(3)}, \boldsymbol{\gamma}^{(4)} \in G_{j}} h\left(\boldsymbol{\gamma}^{(1)}\right) \cdots h\left(\boldsymbol{\gamma}^{(4)}\right) \\
\times \dot{\delta}_{l}(\bar{\gamma}) \mathbf{E}\left[e\left(\sum_{k \in[1, s]} \sum_{\nu \in[1,4]} \theta_{k} \kappa_{k}^{(\nu)} \gamma_{k}^{(\nu)} N_{k} / 2\right)\right] .
\end{array}
$$

Then

$$
\dot{H}_{i, j}(l)=O\left(j^{s-2} n^{4 / 9(s-1)+2 / 45}\right) .
$$

P r o of. Applying (2.4.7) and Lemma 6, we get

$$
\begin{aligned}
\ddot{H}_{i, j}(l)=O( & \sum_{\kappa^{(1)}, \ldots, \kappa^{(4)} \in\{-1,1\}^{s}} \sum_{\gamma^{(1)}, \gamma^{(2)} \in \dot{G}_{i}} \sum_{\gamma^{(3)}, \boldsymbol{\gamma}^{(4)} \in \dot{G}_{j}} \\
& \left.\times\left|\operatorname{Nm}\left(\gamma^{(1)} \ldots \gamma^{(4)}\right)\right|^{-1} \dot{\delta}_{l}(\bar{\gamma}) \prod_{\nu \in[1, s]} \min \left(1, \frac{1}{N_{\nu}\left|\dot{\gamma}_{\nu}\right|}\right)\right) .
\end{aligned}
$$

We will prove Lemma [16 separately for each $l \in[2,7]$ :

Case $l \in\{2,5\}$ : We will consider the case $l=2$. The proof for the case $l=5$ is similar. By (2.7.1) and (2.7.9), we have

with

$$
\dot{H}_{i, j}(2)=O\left(\sum_{1 \leq \nu \leq s} \tilde{H}_{i, j, \nu}\right) .
$$

$$
\tilde{H}_{i, j, \nu}=\sum_{\boldsymbol{\kappa}^{(1)}, \ldots, \boldsymbol{\kappa}^{(4)} \in\{-1,1\}^{s}} \sum_{\boldsymbol{\gamma}^{(1)}, \boldsymbol{\gamma}^{(2)} \in \dot{G}_{i}} \sum_{\boldsymbol{\gamma}^{(3)}, \boldsymbol{\gamma}^{(4)} \in G_{j}} \frac{\left|\operatorname{Nm}\left(\boldsymbol{\gamma}^{(1)} \cdots \boldsymbol{\gamma}^{(4)}\right)\right|}{\delta\left(\dot{\gamma}_{\nu}=0, \ddot{\gamma}_{\nu} \neq 0\right)} .
$$

Let

$$
\gamma^{(j)}=\tilde{\gamma}^{(j)} \cdot \sigma\left(\eta^{(j)}\right) \quad \text { with } \quad \tilde{\gamma}^{(j)} \in \mathbb{F}_{n}
$$

and

$\eta^{(j)} \in \dot{\mathfrak{U}}\left(\gamma^{(j)}, a_{j}, b_{j}\right)(j=1, \ldots, 4), a_{1}=a_{2}=i n^{4 / 9}, a_{3}=a_{4}=j n^{4 / 9}$

and

$$
b_{1}=\cdots=b_{4}=n^{4 / 9}-n^{2 / 9}
$$




\section{GAUSSIAN LIMITING DISTRIBUTION OF LATTICE POINTS IN PARALLEPIDED}

We fix $\tilde{\gamma}^{(1)}, \tilde{\gamma}^{(2)}, \tilde{\gamma}^{(3)}, \tilde{\gamma}^{(4)}$ and $\eta^{(4)}$. Bearing in mind that $\ddot{\gamma}_{\nu} \neq 0$ and $i<j$, we obtain that there is no degenerate solutions $\left(\eta^{(1)}, \eta^{(2)}, \eta^{(3)}\right)$ (see (2.3.1)) of the equation

$$
\dot{\gamma}_{\nu}=\sum_{1 \leq k \leq 4} \kappa_{\nu}^{(k)} \tilde{\gamma}_{\nu}^{(k)} \sigma_{\nu}\left(\eta^{(k)}\right)=0 .
$$

Similarly to (2.6.8) and (2.6.9), we derive from Theorem A, 2.2.11) and (2.2.12) that

$$
\tilde{H}_{i, j, \nu}=O\left(\sum_{\substack{\tilde{\boldsymbol{\gamma}}^{(j)} \in \mathbb{F}_{n} \\ 1 \leq j \leq 4}} \sum_{\boldsymbol{\eta}^{(4)} \in \dot{\mathfrak{U}}\left(\tilde{\boldsymbol{\gamma}}^{(4)}, a_{4}, b_{4}\right)} \frac{1}{\operatorname{Nm}\left(\tilde{\boldsymbol{\gamma}}^{(j)}\right)}\right)=O\left(j^{s-2} n^{4 / 9(s-1)}(\ln n)^{4}\right) .
$$

Hence, the assertion (2.7.8) is proved.

Case $l \in\{3,7\}$ : We have from (2.7.1) for both cases $l=3$ and $l=7$ that there exists $\nu \in[1, s]$ such that $\dot{\gamma}_{\nu} \neq 0, \ddot{\gamma}_{\nu}=0$ and $\dot{\gamma}_{\nu}=\kappa_{\nu}^{(1)} \gamma_{\nu}^{(1)}+\kappa_{\nu}^{(2)} \gamma_{\nu}^{(2)}$. Applying Lemma 4, we get $\left|N_{\nu} \dot{\gamma}_{\nu}\right| \geq \dot{c} n^{20 s}$. Now using (2.7.5), (2.7.6) and (2.7.9), we obtain (2.7.8).

Case $l=4$ : By (2.7.1), we have that there exist $\mu, \nu \in[1, s]$ with $\ddot{\gamma}_{\nu}=0$, $\dot{\gamma}_{\nu}=0$, and $\dot{\gamma}_{\mu} \neq 0$. It is easy to derive that $\gamma^{(1)}= \pm \gamma^{(2)}, \gamma^{(3)}= \pm \gamma^{(4)}$ and $\dot{\gamma}_{\mu}=\tilde{\kappa}_{1} \gamma_{\mu}^{(1)}+\tilde{\kappa}_{2} \gamma_{\mu}^{(3)}$ with $\tilde{\kappa}_{i} \in\{-2,0,2\}, i=1,3$. Hence

$$
\left|\dot{\gamma}_{\mu}\right|=2\left|\gamma_{\mu}^{(1)}\right| \quad \text { or } \quad\left|\dot{\gamma}_{\mu}\right|=2\left|\gamma_{\mu}^{(3)}\right| \quad \text { or } \quad\left|\dot{\gamma}_{\mu}\right|=2\left|\gamma_{\mu}^{(1)} \pm \gamma_{\mu}^{(3)}\right| \neq 0 \text {. }
$$

Applying (2.3.4) and Lemma 4, we get $\left|N_{\mu} \dot{\gamma}_{\mu}\right| \geq \dot{c} n^{20 s}$ for sufficiently large $N$. By (2.7.5), (2.7.6) and (2.7.9), we obtain (2.7.8).

Case $l=6$ : By (2.3.8), we have that there exists $\nu \in[1, s]$ such that $\gamma_{\nu}^{(4)} \geq 2^{j n^{4 / 9}}$. Using Lemma 4, we obtain for sufficiently large $N$ that

$$
\begin{gathered}
\left|\ddot{\gamma}_{\nu}\right|=\left|\kappa_{\nu}^{(3)} \gamma_{\nu}^{(3)}+\kappa_{\nu}^{(4)} \gamma_{\nu}^{(4)}\right| \geq\left|\gamma_{\nu}^{(4)}\right| \exp \left(-\ddot{c}(\ln n)^{3}\right) \geq 2^{j n^{4 / 9}} \exp \left(-\ddot{c}(\ln n)^{3}\right) \mid \\
\geq 2^{(i+1) n^{4 / 9}-n^{2 / 9}+2} \geq 2\left|\kappa_{\nu}^{(1)} \gamma_{\nu}^{(1)}+\kappa_{\nu}^{(2)} \gamma_{\nu}^{(2)}\right|
\end{gathered}
$$

Hence, we get for sufficiently large $N$ that

$$
N_{\nu}\left|\dot{\gamma}_{\nu}\right|=N_{\nu}\left|\kappa_{\nu}^{(1)} \gamma_{\nu}^{(1)}+\kappa_{\nu}^{(2)} \gamma_{\nu}^{(2)}+\kappa_{\nu}^{(3)} \gamma_{\nu}^{(3)}+\kappa_{\nu}^{(4)} \gamma_{\nu}^{(4)}\right| \geq N_{\nu}\left|\ddot{\gamma}_{\nu}\right| / 2 \geq n^{20 s}
$$

Now from (2.7.5), (2.7.6) and (2.7.9), we obtain (2.7.8). Thus, Lemma 16 is proved.

Let

$$
H_{i, j}=\sum_{1 \leq l \leq 7}\left(\ddot{H}_{i, j}(l)-\dot{H}_{i, j}(l)\right) .
$$


By (2.7.2), (2.7.3) and (2.7.7), we get

$$
\begin{aligned}
H_{i, j}= & \sum_{\boldsymbol{\kappa}^{(1)}, \ldots, \boldsymbol{\kappa}^{(4)} \in\{-1,1\}^{s}} \sum_{\boldsymbol{\gamma}^{(1)}, \boldsymbol{\gamma}^{(2)} \in \dot{G}_{i}} \sum_{\boldsymbol{\gamma}^{(3)}, \boldsymbol{\gamma}^{(4)} \in G_{j}} h\left(\boldsymbol{\gamma}^{(1)}\right) \cdots h\left(\boldsymbol{\gamma}^{(4)}\right) \\
& \times\left(\mathbf{E}\left[e\left(\sum_{k \in[1, s]} \sum_{l \in[1,4]} \phi_{k, l}\right)\right]-\mathbf{E}\left[e\left(\sum_{k \in[1, s]} \sum_{l \in[1,2]} \phi_{k, l}\right)\right] \mathbf{E}\left[e\left(\sum_{k \in[1, s]} \sum_{l \in[3,4]} \phi_{k, l}\right)\right]\right)
\end{aligned}
$$

with $\phi_{k, l}=\theta_{k} \kappa_{k}^{(l)} \gamma_{k}^{(l)} N_{k} / 2$.

LEMмA 17. With the notations as above, we have

$$
\varkappa:=\mathbf{E}\left[\left(\sum_{i \in\left[1, n^{5 / 9}\right]}\left(\mathcal{A}^{2}\left(\dot{G}_{i}\right)-\mathbf{E}\left[\mathcal{A}^{2}\left(\dot{G}_{i}\right)\right]\right)\right)^{2}\right]=O\left(n^{2(s-1)-2 / 5}\right) .
$$

Proof. Let

$$
\varkappa_{i, j}=\mathbf{E}\left[\left(\mathcal{A}^{2}\left(\dot{G}_{i}\right)-\mathbf{E}\left[\mathcal{A}^{2}\left(\dot{G}_{i}\right)\right]\right) \times\left(\mathcal{A}^{2}\left(\dot{G}_{j}\right)-\mathbf{E}\left[\mathcal{A}^{2}\left(\dot{G}_{j}\right)\right]\right)\right] .
$$

It is easy to see that

$$
\varkappa_{i, j}=\mathbf{E}\left[\mathcal{A}^{2}\left(\dot{G}_{i}\right) \mathcal{A}^{2}\left(\dot{G}_{j}\right)\right]-\mathbf{E}\left[\mathcal{A}^{2}\left(\dot{G}_{i}\right)\right] \mathbf{E}\left[\mathcal{A}^{2}\left(\dot{G}_{j}\right)\right],
$$

and

$$
\varkappa \leq \dot{\varkappa}+\ddot{\varkappa}, \quad \text { with } \dot{\varkappa}=\sum_{i \in\left[0, n^{5 / 9}\right]} \varkappa_{i, i} \text { and } \ddot{\varkappa}=2 \sum_{i, j \in\left[1, n^{5 / 9}\right], i<j}\left|\varkappa_{i, j}\right| .
$$

By Lemma 14, we obtain

$$
\varkappa_{i, i} \leq \mathbf{E}\left[\left|\mathcal{A}\left(\dot{G}_{i}\right)\right|^{4}\right]=O\left(i^{2(s-2)} n^{8 / 9(s-1)}\right),
$$

and

$$
\dot{\varkappa}=O\left(\sum_{i \in\left[0, n^{5 / 9}\right]} i^{2(s-2)} n^{8 / 9(s-1)}\right)=O\left(n^{2(s-1)-5 / 9}\right) .
$$

Using (2.4.9) and (2.7.11), we get

$$
\varkappa_{i, j}=H_{i, j} .
$$

From (2.7.1), 2.7.3) and (2.7.7), we derive

$$
\ddot{H}_{i, j}(1)-\dot{H}_{i, j}(1)=0 .
$$

By Lemma 15 and Lemma 16, we have

$$
\dot{H}_{i, j}(l)=O\left(n^{-10 s}\right) \text { and } \ddot{H}_{i, j}(l)=O\left(j^{s-2} n^{4 / 9(s-1)+2 / 45}\right), l=2,3, \ldots, 7, i<j \text {. }
$$


Applying (2.7.10), we obtain $H_{i, j}=O\left(j^{s-2} n^{4 / 9(s-1)}\right)$. Now from (2.7.13) and (2.7.15), we get

$$
\ddot{\varkappa}=O\left(\sum_{j \in\left[1, n^{5 / 9}\right]} j^{s-1} n^{4 / 9(s-1)+2 / 45}\right)=O\left(n^{5 s / 9+(s-1) 4 / 9+2 / 45}\right)=O\left(n^{s-2 / 5}\right) .
$$

By (2.7.13) and (2.7.14), Lemma 17 is proved.

\subsection{Martingale approximation}

Denote by $\dot{\mathcal{F}}(l)$ the sigma field on $[0,1)^{s}$ generated by

$$
\left\{\left[\frac{k_{1}}{2^{l}}, \frac{k_{1}+1}{2^{l}}\right) \times \cdots \times\left[\frac{k_{s}}{2^{l}}, \frac{k_{s}+1}{2^{l}}\right): k_{1}, \ldots, k_{s}=0, \ldots, 2^{l}-1\right\} .
$$

Let

$$
l(0)=0, l(i)=(i+1)\left[n^{4 / 9}\right]+\left[n / s-n^{1 / 9}\right],
$$

$$
\mathcal{F}_{i}=\dot{\mathcal{F}}(l(i)) \quad \text { and } \quad \xi_{i}=\mathbf{E}\left[\mathcal{A}\left(\dot{G}_{i}\right) \mid \mathcal{F}_{i}\right]-\mathbf{E}\left[\mathcal{A}\left(\dot{G}_{i}\right) \mid \mathcal{F}_{i-1}\right], i=1,2, \ldots
$$

Then $\left(\xi_{i}\right)_{i \geq 1}$ is the martingale difference array satisfying

$$
\mathbf{E}\left[\xi_{i} \mid \mathcal{F}_{i-1}\right]=0, \quad i=1,2, \ldots
$$

LEMMA 18. With the notations as above

$$
\begin{aligned}
& \mathbf{E}\left[\mathcal{A}\left(\dot{G}_{i}\right) \mid \mathcal{F}_{i-1}\right]=O\left(n^{-10 s}\right), \quad \mathcal{A}\left(\dot{G}_{i}\right)-\xi_{i}=O\left(n^{-10 s}\right), \\
& \mathcal{A}\left(\dot{G}_{i}\right)^{2}-\xi_{i}^{2}=O\left(n^{-8 s}\right) \quad \text { and } \quad\left|\xi_{i}\right|^{4} \leq 8\left|\mathcal{A}\left(\dot{G}_{i}\right)\right|^{4}+O\left(n^{-6 s}\right) .
\end{aligned}
$$

Proof. It is easy to see that

$$
\left|2^{l} \int_{k / 2^{l}}^{(k+1) / 2^{l}} \sin (\lambda \theta) \mathrm{d} \theta\right|=2^{\mathrm{l}} / \lambda\left|\cos \left(\lambda(\mathrm{k}+1) / 2^{\mathrm{l}}\right)-\cos \left(\lambda \mathrm{k} / 2^{\mathrm{l}}\right)\right| \leq 2^{\mathrm{l}+1} / \lambda \quad \text { with } \lambda>0 .
$$

Hence, we obtain for $\left|\gamma_{j}\right| \geq 2^{i\left[n^{4 / 9}\right]}$ and $\left|N_{j}\right| \geq c_{0}^{-1} 2^{(n-1) / s}$ that

$$
\left|2^{l_{i-1}} \int_{k / 2^{l_{i-1}}}^{(k+1) / 2^{l_{i-1}}} \sin \left(N_{j} \gamma_{j} \theta\right) \mathrm{d} \theta\right| \leq \mathrm{c}_{0} 2^{-\left[\mathrm{n}^{1 / 9}\right]+5} .
$$

Bearing in mind that

$$
\mathbf{E}\left[\phi_{1}\left(\theta_{1}\right) \cdots \phi_{s}\left(\theta_{s}\right) \mid \mathcal{F}_{i-1}\right]=\prod_{j=1}^{s} 2^{l_{i}} \int_{k_{j} / 2^{l_{i-1}}}^{\left(k_{j}+1\right) / 2^{l_{i-1}}} \phi_{j}\left(\theta_{j}\right) \mathrm{d} \theta_{\mathrm{j}}
$$

on $\left[\frac{k_{1}}{2^{l}-1}, \frac{k_{1}+1}{2^{l_{i-1}}}\right) \times \cdots \times\left[\frac{k_{s}}{2^{l^{i}-1}} \frac{k_{s}+1}{2^{l_{i-1}}}\right)$, we have from (2.3.8), (2.1.2), (2.4.1), Lemma 3 and (2.8.4), that

$$
\mathbf{E}\left[\mathcal{A}\left(\dot{G}_{i}\right) \mid \mathcal{F}_{i-1}\right]=O\left(n^{s} 2^{-\left[n^{1 / 9}\right]}\right)=O\left(n^{-10 s}\right) .
$$


Now let

$$
\left|\gamma_{j}\right| \leq 2^{(i+1)\left[n^{4 / 9}\right]-\left[n^{2 / 9}\right]} \quad \text { and } \quad \theta_{j}^{(1,2)} \in\left[\frac{k}{2^{l_{i}}}, \frac{k+1}{2^{l_{i}}}\right)
$$

then

$$
\begin{aligned}
\left|\sin \left(N_{j} \gamma_{j} \theta_{j}^{(1)}\right)-\sin \left(N_{j} \gamma_{j} \theta_{j}^{(2)}\right)\right| & =\left|N_{j} \gamma_{j}\left(\theta_{j}^{(1)}-\theta_{j}^{(2)}\right) \cos \left(N_{j} \gamma_{j} \theta_{j}^{(3)}\right)\right| \\
& \leq 2^{\left[n^{1 / 9}\right]-\left[n^{2 / 9}\right]+2} c_{0},
\end{aligned}
$$

with

$$
\theta_{j}^{(3)} \in\left[\frac{k}{2^{l_{i}}}, \frac{k+1}{2^{l_{i}}}\right) .
$$

Therefore

$$
\prod_{j=1}^{s} \sin \left(N_{j} \gamma_{j} \theta_{j}^{(1)}\right)=\prod_{j=1}^{s} \sin \left(N_{j} \gamma_{j} \theta_{j}^{(2)}\right)+O\left(2^{\left[n^{1 / 9}\right]}-\left[n^{2 / 9}\right]\right) .
$$

Thus

$$
\prod_{j=1}^{s} \sin \left(N_{j} \gamma_{j} \theta_{j}\right)=\mathbf{E}\left[\prod_{j=1}^{s} \sin \left(N_{j} \gamma_{j} \theta\right) \mid \mathcal{F}_{i}\right]+O\left(2^{\left[n^{1 / 9}\right]-\left[n^{2 / 9}\right]}\right) .
$$

Taking into account (2.3.8), (2.1.2), (2.4.1) and (2.8.5), we get (2.8.2). It is easy to see that

$$
\begin{aligned}
\left|\mathcal{A}\left(\dot{G}_{i}\right)^{2}-\xi_{i}^{2}\right| & \leq\left(2\left|\mathcal{A}\left(\dot{G}_{i}\right)\right|+\left|\mathcal{A}\left(\dot{G}_{i}\right)-\xi_{i}\right|\right)\left|\mathcal{A}\left(\dot{G}_{i}\right)-\xi_{i}\right|, \\
\left|\xi_{i}\right|^{4} & \leq 8\left|\mathcal{A}\left(\dot{G}_{i}\right)\right|^{4}+8\left|\mathcal{A}\left(\dot{G}_{i}\right)-\xi_{i}\right|^{4} .
\end{aligned}
$$

Applying (2.4.13), we obtain (2.8.3). Hence, Lemma 18 is proved.

We shall use the following variant of the martingale central limit theorem (see [Mo, p. 414]):

Let $(\Omega, \mathcal{F}, P)$ be a probability space and $\left\{\left(\zeta_{n, k}, F_{n, k}\right) \mid n=1,2, \ldots, k=\right.$ $\left.1, \ldots, k_{n}\right\}$ be a martingale difference array with $\mathbf{E}\left[\zeta_{n, k} \mid F_{n, k-1}\right]=0$ a.s. $\left(F_{n, 0}\right.$ is the trivial field).

Theorem C. Let $L(n, \epsilon)=\sum_{1 \leq k \leq k_{n}} \mathbf{E}\left[\zeta_{n, k}^{2} \delta\left(\left|\zeta_{n, k}\right|>\epsilon\right)\right]$,

$$
\begin{gathered}
\mathbb{S}_{n}=\sum_{1 \leq k \leq i} \zeta_{n, k}, \quad \text { and } \quad \mathbb{V}_{n}^{2}=\sum_{1 \leq k \leq k_{n}} \mathbf{E}\left[\zeta_{n, k}^{2} \mid F_{n, k-1}\right], \\
\mathbb{A}_{n}=\mathbf{E}\left[\left|\mathbb{V}_{n}^{2}-1\right|\right], \quad \mathbb{W}_{n}=\int_{0}^{1} L(n, \epsilon) \mathrm{d} \epsilon, \quad \text { and } \sum_{1 \leq \mathrm{k} \leq \mathrm{k}_{\mathrm{n}}} \mathbf{E}\left[\zeta_{\mathrm{n}, \mathrm{k}}^{2}\right]=1 .
\end{gathered}
$$

Then

$$
\sup _{t}\left|P\left(\mathbb{S}_{n}<t\right)-\Phi(t)\right| \leq 7\left(\mathbb{W}_{n}^{1 / 4}+\mathbb{A}_{n}^{1 / 3}\right)
$$


Now we apply Theorem $\mathrm{C}$ to the martingale difference array (2.8.1) with $F_{n, k}=\mathcal{F}_{k}, \zeta_{n, i}=\xi_{i} / \varrho, \varrho=\left(\sum_{i \in\left[1, k_{n}\right]} \mathbf{E}\left[\xi_{i}^{2}\right]\right)^{1 / 2}$, and $k_{n}=\left[n^{5 / 9}\right]$.

Lemma 19. Let

$$
\mathbb{S}_{n}=\sum_{1 \leq i \leq k_{n}} \xi_{i} / \varrho
$$

Then

$$
\sup _{t}\left|P\left(\mathbb{S}_{n}<t\right)-\Phi(t)\right|=O\left(n^{-1 / 15}\right)
$$

P r o of. By (2.8.1), $\left(\xi_{i}\right)_{i \geq 1}$ is the martingale difference sequence (and consequently orthogonal). Using the triangle inequality, Lemma 11, Lemma 13 and Lemma 18, we obtain

$$
\begin{aligned}
\varrho^{2} & =\sum_{i \in\left[1, k_{n}\right]} \mathbf{E}\left[\xi_{i}^{2}\right]=\mathbf{E}\left[\left(\sum_{i \in\left[1, k_{n}\right]} \xi_{i}\right)^{2}\right]=\mathbf{E}\left[\sum_{i \in\left[1, k_{n}\right]} \mathcal{A}\left(\dot{G}_{i}\right)^{2}\right]+O(1) \\
& =\mathbf{E}\left[\left(\mathcal{R}\left(\boldsymbol{\theta} \cdot \mathbf{N} \cdot \mathbb{K}^{s}+\mathbf{x}, \Gamma\right)\right)^{2}\right]+O\left(n^{s-1-2 / 9}\right) \in n^{s-1}\left[w_{3}, w_{4}\right],
\end{aligned}
$$

with some $w_{4}>w_{3}>0$.

Let $\dot{\mathcal{F}}$ be a sub- $\sigma$-algebra of $\mathcal{F}$. By Jensen's inequality, we get

$$
\mathbf{E}\left[|\vartheta|^{\alpha}\right] \leq\left(\mathbf{E}\left[|\vartheta|^{\beta}\right]\right)^{\alpha / \beta} \text { and } \quad \mathbf{E}\left[|\vartheta|^{\alpha} \mid \dot{\mathcal{F}}\right] \leq\left(\mathbf{E}\left[|\vartheta|^{\beta} \mid \dot{\mathcal{F}}\right]\right)^{\alpha / \beta} \text {, with } \beta>\alpha>0 \text {. }
$$

Consider $\mathbb{W}_{n}$. We derive from (2.8.8) that

$$
\begin{aligned}
\mathbb{W}_{n}= & \sum_{1 \leq i \leq k_{n}} \int_{0}^{1} \int\left|\xi_{i} / \varrho\right|^{2} \delta\left(\xi_{i} / \varrho \mid>\epsilon\right) \mathrm{dP} \mathrm{d} \epsilon \leq \sum_{1 \leq \mathrm{i} \leq \mathrm{k}_{\mathrm{n}}} \int_{0}^{1} \int_{\left\{\left|\xi_{\mathrm{i}} / \varrho\right|>\epsilon\right\}}\left|\xi_{\mathrm{i}} / \varrho\right|^{2} \\
& \times\left|\xi_{i} /(\varrho \epsilon)\right|^{24 / 25} \mathrm{dP} \mathrm{d} \epsilon \leq \sum_{1 \leq \mathrm{i} \leq \mathrm{k}_{\mathrm{n}}} \int\left|\xi_{\mathrm{i}} / \varrho\right|^{74 / 25} \mathrm{dP} \int_{0}^{1} \epsilon^{-24 / 25} \mathrm{~d} \epsilon .
\end{aligned}
$$

Applying (2.8.11) and (2.8.12) with $\alpha=74 / 25, \beta=4$, we have

$$
\mathbb{W}_{n} \leq 25 \sum_{i=1}^{k_{n}}\left(\int\left|\xi_{i} / \varrho\right|^{4} \mathrm{dP}\right)^{37 / 50}=O\left(n^{-(s-1) 37 / 25} \sum_{i=1}^{k_{n}}\left(\mathbf{E}\left[\mathcal{A}^{4}\left(\dot{G}_{i}\right)\right]\right)^{37 / 50}\right)
$$


By Lemma 14, we get

$$
\begin{aligned}
\mathbb{W}_{n} & =O\left(n^{-(s-1) 37 / 100} \sum_{1 \leq i \leq k_{n}} i^{2(s-2) \frac{37}{25}} n^{\frac{8}{9}(s-1) \frac{37}{50}}\right) \\
& =O\left(n^{-(s-1) \frac{37}{25}+\frac{5}{9}\left(2(s-2) \frac{37}{50}+1\right)+\frac{8}{9}(s-1) \frac{37}{50}}\right) \\
& =O\left(n^{-4 / 15}\right) \text { and } \mathbb{W}_{n}^{1 / 4}=O\left(n^{-1 / 15}\right) .
\end{aligned}
$$

Next consider $\mathbb{A}_{n}$. Let

$$
\mathbb{U}_{n}^{2}=\sum_{1 \leq k \leq k_{n}}\left(\xi_{i} / \varrho\right)^{2} .
$$

Using (2.8.11), 2.8.15) and Lemma 18, we derive

$$
\begin{aligned}
\mathbf{E}\left[\left|\mathbb{U}_{n}^{2}-1\right|^{2}\right] & =\varrho^{-4} \mathbf{E}\left[\left|\sum_{1 \leq k \leq k_{n}}\left(\xi_{i}^{2}-\mathbf{E}\left[\xi_{i}^{2}\right]\right)\right|^{2}\right] \\
& \leq 2 \varrho^{-4} \mathbf{E}\left[\left(\sum_{1 \leq k \leq k_{n}}\left(\mathcal{A}^{2}\left(\dot{G}_{i}\right)-\mathbf{E}\left[\mathcal{A}^{2}\left(\dot{G}_{i}\right)\right]\right)\right)^{2}\right]+O\left(n^{-5}\right) .
\end{aligned}
$$

By Lemma 17, we obtain

$$
\mathbf{E}\left[\left|\mathbb{U}_{n}^{2}-1\right|^{2}\right]=O\left(n^{-2(s-1)+2(s-1)-5 / 9}\right)=O\left(n^{-5 / 9}\right) .
$$

Let

$$
\varsigma_{i}=\left(\xi_{i} / \varrho\right)^{2}-\mathbf{E}\left[\left(\xi_{i} / \varrho\right)^{2} \mid \mathcal{F}_{i-1}\right] \quad \text { and } \quad \mathbb{V}_{n}^{2}=\sum_{1 \leq i \leq k_{n}} \mathbf{E}\left[\left(\xi_{i} / \varrho\right)^{2} \mid \mathcal{F}_{i-1}\right]
$$

By (2.8.1), we see that $\left(\varsigma_{i}\right)_{i \geq 1}$ is the martingale difference array satisfying $\mathbf{E}\left[\varsigma_{i} \mid \mathcal{F}_{i-1}\right]=0, i=1,2, \ldots$ From (2.8.15), we have

$$
\mathbf{E}\left[\left|\mathbb{V}_{n}^{2}-\mathbb{U}_{n}^{2}\right|^{2}\right]=\mathbf{E}\left[\left|\sum_{1 \leq i \leq k_{n}} \varsigma_{i}\right|^{2}\right]=\sum_{1 \leq i \leq k_{n}} \mathbf{E}\left[\varsigma_{i}^{2}\right]
$$

Using (2.8.18) and (2.8.12), we get

$$
\mathbf{E}\left[\varsigma_{i}^{2}\right] \leq 2 \varrho^{-4}\left(\mathbf{E}\left[\xi_{i}^{4}\right]+\mathbf{E}\left[\left(\mathbf{E}\left[\xi_{i}^{2} \mid \mathcal{F}_{i-1}\right]\right)^{2}\right]\right) \leq 4 \varrho^{-4} \mathbf{E}\left[\xi_{i}^{4}\right] .
$$


By Lemma 14, Lemma 18 and (2.8.11), we have

$$
\begin{aligned}
\mathbf{E}\left[\left|\mathbb{V}_{n}^{2}-\mathbb{U}_{n}^{2}\right|^{2}\right] & =O\left(n^{-2(s-1)} \sum_{i=1}^{k_{n}} \mathbf{E}\left[\mathcal{A}^{4}\left(\dot{G}_{i}\right)\right]+n^{-7 s}\right) \\
& =O\left(n^{-2(s-1)} \sum_{i=1}^{k_{n}} i^{2(s-2)} \times n^{8 / 9(s-1)}\right) \\
& =O\left(n^{-2(s-1)+5 / 9(2 s-3)+8 / 9(s-1)}\right)=O\left(n^{-5 / 9}\right) .
\end{aligned}
$$

By (2.8.8) and (2.8.12), we get

$$
\begin{aligned}
\mathbb{A}_{n}^{2} & =\left(\mathbf{E}\left[\left|\mathbb{V}_{n}^{2}-1\right|\right]\right)^{2} \leq \mathbf{E}\left[\left|\mathbb{V}_{n}^{2}-1\right|^{2}\right] \\
& =\mathbf{E}\left[\left|\mathbb{V}_{n}^{2}-\mathbb{U}_{n}^{2}+\mathbb{U}_{n}^{2}-1\right|^{2}\right] \\
& \leq 2 \mathbf{E}\left[\left|\mathbb{V}_{n}^{2}-\mathbb{U}_{n}^{2}\right|^{2}\right]+2 \mathbf{E}\left[\left|\mathbb{U}_{n}^{2}-1\right|^{2}\right]
\end{aligned}
$$

From (2.8.17) and (2.8.19), we derive

$$
\mathbb{A}_{n}^{2}=O\left(n^{-2 / 5}\right), \quad \text { and } \quad \mathbb{A}_{n}^{1 / 3}=O\left(n^{-1 / 15}\right) .
$$

Applying (2.8.14) and Theorem C, we obtain the assertion of Lemma 19,

\subsection{End of the proof of Theorem 1}

Let $\dot{\mathbb{S}}_{n}=\mathcal{R}\left(\boldsymbol{\theta} \cdot \mathbf{N} \cdot \mathbb{K}_{s}+\mathbf{x}, \Gamma\right) / \grave{\varrho}$ and $\dot{\varrho}=\left(\mathbf{E}\left[\mathcal{R}^{2}\left(\boldsymbol{\theta} \cdot \mathbf{N} \cdot \mathbb{K}_{s}+\mathbf{x}, \Gamma\right)\right]\right)^{1 / 2}$. Using Lemma 11 and Lemma [18, we obtain

$$
\begin{aligned}
& \mathbf{E}\left[\left(\mathcal{R}\left(\boldsymbol{\theta} \cdot \mathbf{N} \cdot \mathbb{K}_{s}+\mathbf{x}, \Gamma\right)-\sum_{1 \leq i \leq k_{n}} \xi_{i}\right)^{2}\right] \\
& \leq 2 \mathbf{E}\left[\left(\mathcal{R}\left(\boldsymbol{\theta} \cdot \mathbf{N} \cdot \mathbb{K}_{s}+\mathbf{x}, \Gamma\right)-\sum_{1 \leq i \leq k_{n}} \mathcal{A}\left(\dot{G}_{i}\right)\right)^{2}\right] \\
& +2 \mathbf{E}\left[\left(\sum_{1 \leq i \leq k_{n}}\left(\mathcal{A}\left(\dot{G}_{i}\right)-\xi_{i}\right)\right)^{2}\right]=O\left(n^{s-1-2 / 9}\right) .
\end{aligned}
$$

By (2.8.11), we get $\dot{\varrho}^{2}-\varrho^{2}=O\left(n^{s-1-2 / 9}\right), \dot{\varrho}^{2} \geq w_{2} n^{s-1}$ for some $w_{2}>0$, and

$$
\left|\frac{1}{\varrho}-\frac{1}{\dot{\varrho}}\right|=\frac{|\varrho-\dot{\varrho}|}{\varrho \dot{\varrho}}=\frac{\left|\varrho^{2}-\dot{\varrho}^{2}\right|}{\varrho \dot{\varrho}|\varrho+\dot{\varrho}|}=O\left(n^{-3 / 2(s-1)-2 / 9}\right) \text {. }
$$


Applying (2.8.10), (2.8.11), (2.9.2) and (2.9.1), we derive

$$
\begin{aligned}
\mathbf{E}\left[\left(\mathbb{S}_{n}-\dot{\mathbb{S}}_{n}\right)^{2}\right] \leq 2 \mathbf{E}[ & \left.\left(\sum_{1 \leq k \leq k_{n}} \xi_{i}-\mathcal{R}\left(\boldsymbol{\theta} \cdot \mathbf{N} \cdot \mathbb{K}_{s}+\mathbf{x}, \Gamma\right)\right)^{2} / \varrho^{2}\right] \\
& +2(1 / \varrho-1 / \varrho)^{2} \mathbf{E}\left[\mathcal{R}^{2}\left(\boldsymbol{\theta} \cdot \mathbf{N} \cdot \mathbb{K}_{s}+\mathbf{x}, \Gamma\right)\right]=O\left(n^{-2 / 9}\right) .
\end{aligned}
$$

By Chebyshev's inequality, we have

$$
P\left(\left|\dot{\mathbb{S}}_{n}-\mathbb{S}_{n}\right| \geq n^{-1 / 15}\right)=O\left(n^{-2 / 9+2 / 15}\right)=O\left(n^{-1 / 15}\right) .
$$

It is easy to see that

$$
\left\{\dot{\mathbb{S}}_{n}<t\right\} \subseteq\left(\left\{\mathbb{S}_{n}<t+n^{-1 / 15}\right\} \cap\left\{\left|\dot{\mathbb{S}}_{n}-\mathbb{S}_{n}\right| \leq n^{-1 / 15}\right\}\right) \cup\left\{\left|\dot{\mathbb{S}}_{n}-\mathbb{S}_{n}\right| \geq n^{-1 / 15}\right\}
$$

and

$\left\{\mathbb{S}_{n}<t-n^{-1 / 15}\right\} \subseteq\left(\left\{\dot{\mathbb{S}}_{n}<t\right\} \cap\left\{\left|\dot{\mathbb{S}}_{n}-\mathbb{S}_{n}\right| \leq n^{-1 / 15}\right\}\right) \cup\left\{\left|\dot{\mathbb{S}}_{n}-\mathbb{S}_{n}\right| \geq n^{-1 / 15}\right\}$

Hence

$$
\begin{aligned}
& P\left(\left\{\mathbb{S}_{n}<t-n^{-1 / 15}\right\}\right)-P\left(\left\{\left|\dot{\mathbb{S}}_{n}-\mathbb{S}_{n}\right| \geq n^{-1 / 15}\right\}\right) \\
& \leq P\left(\left\{\dot{\mathbb{S}}_{n}<t\right\}\right) \leq P\left(\left\{\mathbb{S}_{n}<t+n^{-1 / 15}\right\}\right)+P\left(\left\{\left|\dot{\mathbb{S}}_{n}-\mathbb{S}_{n}\right| \geq n^{-1 / 15}\right\}\right)
\end{aligned}
$$

We note for $|u| \leq n^{-1 / 15}$ that

$$
|\Phi(t+u)-\Phi(t)|<\frac{1}{\sqrt{2 \pi}} \int_{t-|u|}^{t+|u|} e^{-u^{2} / 2} \mathrm{du} \leq \frac{1}{\sqrt{2 \pi}} \int_{\mathrm{t}-\mathrm{n}^{-1 / 15}}^{\mathrm{t}+\mathrm{n}^{-1 / 15}} \mathrm{du}=\frac{2}{\sqrt{2 \pi}} \mathrm{n}^{-1 / 15} .
$$

Using Lemma 19, we get

$$
\begin{aligned}
& \sup _{t}\left|P\left(\left\{\mathbb{S}_{n}<t+u\right\}\right)-\Phi(t)\right| \\
& \leq \sup _{t}\left(\left|P\left(\left\{\mathbb{S}_{n}<t+u\right\}\right)-\Phi(t+u)\right|+|\Phi(t+u)-\Phi(t)|\right) \\
&=O\left(n^{-1 / 15}\right), \quad|u| \leq n^{-1 / 15}
\end{aligned}
$$

By (2.9.4) and (2.9.3), we derive

$$
\sup _{t}\left|P\left(\dot{\mathbb{S}}_{n}<t\right)-\Phi(t)\right|=O\left(n^{-1 / 15}\right) .
$$

Bearing in mind that throughout the paper $O$-constants does not depend on $\mathbf{x}$, we obtain the assertion of Theorem 1 . 


\section{GAUSSIAN LIMITING DISTRIBUTION OF LATTICE POINTS IN PARALLEPIDED}

\subsection{Sketch of the proof of Theorem 2}

We use notations from $\S 1.3$. Let

$$
\begin{aligned}
I_{0}= & {\left[0, y_{1}\right) \times \cdots \times\left[0, y_{s-1}\right), } \\
I_{1}= & {\left[-y_{1} / 2, y_{1} / 2\right) \times \cdots \times\left[-y_{s-1} / 2, y_{s-1} / 2\right), } \\
I_{2}= & {[-1 / 2,1 / 2)^{s-1}, } \\
I_{3}= & {\left[-y_{s} N \operatorname{det} \Gamma / 2, y_{s} N \operatorname{det} \Gamma / 2\right), } \\
I_{4}= & {\left[-z_{s}\left(\mathbf{x},\left[y_{s} N\right]\right) / 2, z_{s}\left(\mathbf{x},\left[y_{s} N\right]\right) / 2\right), } \\
\mathbf{u}_{1}= & \left(y_{1} / 2, \cdots, y_{s-1} / 2, z_{s}\left(\mathbf{x},\left[y_{s} N\right]\right) / 2\right)-\dot{\mathbf{x}}, \\
\mathbf{u}_{2}= & \left(1 / 2, \cdots, 1 / 2, z_{s}\left(\mathbf{x},\left[y_{s} N\right]\right) / 2\right)-\dot{\mathbf{x}} \\
& \text { with } \mathbf{x}=\left(x_{1}, \cdots, x_{s-1}\right), \dot{\mathbf{x}}=\left(x_{1}, \cdots, x_{s-1}, 0\right) .
\end{aligned}
$$

By (1.1), (1.5) and (1.7), we obtain

$$
\Delta\left(I_{0},\left(\mathcal{T}^{l}(\mathbf{x})\right)_{l=0}^{\left[y_{s} N\right]-1}\right)=\mathcal{N}\left(I_{1} \times I_{3}+\mathbf{u}_{1}, \Gamma\right)-y_{1} \cdots y_{s-1} \mathcal{N}\left(I_{3} \times I_{2}+\mathbf{u}_{2}, \Gamma\right) .
$$

Let $a=z_{s}\left(\mathbf{x},\left[y_{s} N\right]\right), b=y_{s} N \operatorname{det} \Gamma$, and let

$$
(\kappa, I)=\left\{\begin{array}{lc}
(1,[-a / 2,-b / 2) \cup[b / 2, a / 2), & \text { if } a>b, \\
(-1,[-b / 2,-a / 2) \cup[a / 2, b / 2, & \text { otherwise }
\end{array}\right.
$$

By (1.5) and (1.7), we get

$$
\Delta\left(I_{0},\left(\mathcal{T}^{l}(\mathbf{x})\right)_{l=0}^{\left[y_{s} N\right]-1}\right)=\dot{R}_{1}+\kappa \ddot{R}_{1}-y_{1} y_{2} \cdots y_{s-1}\left(\dot{R}_{2}+\kappa \ddot{R}_{2}\right)
$$

with

$$
\dot{R}_{i}=\mathcal{R}\left(I_{i} \times I_{3}+\mathbf{u}_{k}, \Gamma\right), \quad \text { and } \quad \ddot{R}_{i}=\mathcal{R}\left(I_{i} \times I_{4}+\mathbf{u}_{k}, \Gamma\right), \quad i=1,2 .
$$

It is easy to verify (see also [Le2, p. 86]) that

$$
\ddot{R}_{i}=O\left((\ln (n))^{s-1}\right), \quad i=1,2 .
$$

Thus $\dot{R}_{1}-y_{1} y_{2} \cdots y_{s-1} \dot{R}_{2}$ is the essential part of $\Delta\left(I_{0},\left(\mathcal{T}^{l}(\mathbf{x})\right)_{l=0}^{\left[y_{s} N\right]-1}\right)$. Repeating the proofs of $\S 2.4$, we have the upper bound of the variance of $\dot{R}_{1}-y_{1} \cdots y_{s-1} \dot{R}_{2}$. Using Roth's inequality (1.6), we get the lower bound of the variance $\dot{R}_{1}-y_{1} \cdots y_{s-1} \dot{R}_{2}$. Next repeating the proofs of $\S 2.5-\S 2.8$, we obtain the assertion of Theorem 2 , 
Acknowledgements. I am very grateful to the referee for corrections and suggestions which improved this paper.

\section{REFERENCES}

[BW] BAKER, A.- WUSTHOLZ, G.: Logarithmic Forms and Diophantine Geometry, Cambridge University Press, Cambridge, 2007.

[Be1] BECK, J.: Randomness of $n \sqrt{2} \bmod 1$ and a Ramsey property of the hyperbola. Sets, graphs and numbers (G. Halsz ed. et al.), Sets, graphs and numbers. A birthday salute to Vera T.Ss and Andrs Hajnal. Colloq. Math. Soc. Jnos Bolyai. 60, (1992) 23-66.

[Be2] BECK, J.: Randomness in lattice point problems, Discrete Math. 229 (2001), no. 1-3, 29-55.

[Be3] BECK, J.: Probabilistic Diophantine Approximation: Randomness in Lattice Point Counting, Springer, New York, 2014.

[BC] BECK, J.-CHEN, W. W. L.: Irregularities of Distribution. Cambridge Univ. Press, Cambridge, 1987.

[Bl] BLEHER, P.: Trace formula for quantum integrable systems, lattice-point problem, and small divisors (D. A. Hejhal ed. et al.), in: Emerging Applications of Number Theory. Based on the proceedings of the IMA summer program, Minneapolis, MN, USA, July 15-26, 1996. IMA Vol. Math. Appl. 109 (1999), pp. 1-38.

[BS] BOREVICH, A. I.-SHAFEREVITCH, I. R.: Number Theory, Academic Press, New York, 1966.

[By] BYKOVSKIII, V. A.: On the right order of error of optimal cubature formulas in the spaces with dominating derivation and $L^{2}$ discrepancy of nets, Dalnevost. Science Center of the USSR Acad. of Sciences, Vladivostok, 1985. (In Russian)

[DrTi] DRMOTA, M. - TICHY, R.: Sequences, Discrepancies and Applications, in: Lecture Notes in Mathematics Vol. 1651, Springer-Verlag, Berlin, 1997.

[ESS] EVERTSE, J. H.-SCHLICKEWEI, H. P.-SCHMIDT, W. M.: Linear equations in variables which lie in a multiplicative group, Ann. of Math. (2) 155 (2002), no. 3, 807-836.

[Fr] FROLOV, K. K.: Upper bound of the discrepancy in metric $L_{p}, 2 \leq p<\infty$. Dokl. Akad. Nauk SSSR 252 (1980), no. 4, 805-807 (In Russian); English translation: Soviet Math. Dokl. 21 (1980), no. 3, 840-842.

[GL] GRUBER, P. M.-LEKKERKERKER, C. G.: Geometry of Numbers, NorthHolland, New-York, 1987.

[HuRu] HUGHES, C. P.-RUDNICK, Z.: On the distribution of lattice points in thin annuli, Int. Math. Res. Not. 13 (2004), 637-658. 


\section{GAUSSIAN LIMITING DISTRIBUTION OF LATTICE POINTS IN PARALLEPIDED}

[Le1] LEVIN, M. B.: The multidimensional generalization of J.Beck 'Randomness of $n \sqrt{2} \bmod 1 \ldots$ and a.s. invariance principle for $\mathbb{Z}^{d}$-actions of toral automorphisms, Abstracts of Annual Meeting of the Israel Mathematical Union (2002), http://imu.org.il/Meetings/IMUmeeting2002/ergodic.txt

[Le2] LEVIN, M. B.: On low discrepancy sequences and low discrepancy ergodic transformations of the multidimensional unit cube, Israel J. Math. 178 (2010), 61-106.

[Le3] LEVIN, M. B.: Central Limit Theorem for $\mathbb{Z}_{+}^{d}$-actions by toral endomorphisms, Electronic Journal of Probability 18 (2013), no. 35, 42 pp.

[Le4] LEVIN, M. B.: On the lower bound in the lattice point remainder problem for a parallelepiped, Discrete Comput. Geom. 54 (2015), no. 4, 826-870.

[LeMe] LEVIN, M. B.-MERZBACH, E.: Central limit theorems for the ergodic adding machine, Israel J. Math. 134 (2003), 61-92.

[Ma] MARKLOF, J.: Energy level statistics, lattice point problems, and almost modular functions, Frontiers in Number Theory, Physics, and Geometry. I, Springer, Berlin, 2006, pp. 163-181.

[Mo] MORI, T.: On the rate of convergence in the martingale central limit theorem. Studia Sci. Math. Hungar. 12 (1977), no. 3-4, 413-417.

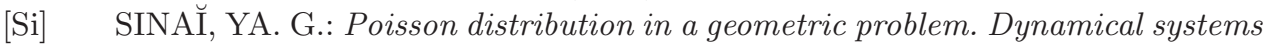
and statistical mechanics (Moscow, 1991), Adv. Soviet Math. 3, Amer. Math. Soc., Providence, RI, 1991. pp. 199-214.

[Skr] SKRIGANOV, M. M.: Construction of uniform distributions in terms of geometry of numbers, Algebra i Analiz 6 (1994), no. 3 200-230; Reprinted in: St. Petersburg Math. J. 6(1995), no. 3 635-664.

[SW] STEIN, E.-WEISS, G.: Introduction to Fourier Analysis on Euclidean Spaces, Princeton University Press, New-York, 1971.

Received September 10, 2015

Accepted October 19, 2015
Mordechay B. Levin

Department of Mathematics

Bar-Ilan University

Ramat-Gan, 52900

ISRAEL

E-mail:mlevin@math.biu.ac.il 